\title{
STRONG INTERACTION EXPERIMENTS AT THE ZGS Tris Coffin
}

The University of Michigan, Ann Arbor, Mi. 48109

\section{ABSTRACT}

The history of two related strong interaction experiments carried out at the Argonne ZGS accelerator in the mid 1960's is described. These experiments involved the study of the production and decay distributions of $\pi+p \rightarrow \rho+N$ for forward production of $\rho$, and $\pi+p \rightarrow N^{*}+\pi$ for backward production of $N^{*}$. Special emphasis is given to the role played by Kent Terwilliger in conceiving these experiments and bringing them to a successful conclusion. A literature search is carried out to assess the impact of these experiments on the strong interaction physics of the period.

\section{INTRODUCTION}

Don Meyer has described several strong interaction experiments that he and Kent and I did during the early 1960's. These experiments dealt with simple two body final states and were studied with a relatively simple spark chamber setup. We collaborated on one final set of strong interaction experiments in the late 60's in which we studied more complicated three body final states with, necessarily, a more complicated apparatus. Specifically, we studied the production and subsequent decay of the $\rho$ meson and the production of resonant states of the nucleon, $N^{*}$, in pion proton interactions;

$$
\begin{array}{ll}
\pi^{-} p \rightarrow \rho N & \text { forward production } \\
\pi^{-} p \rightarrow N^{*} \pi & \text { backward production }
\end{array}
$$

Each of these reactions was restricted to a very narrow kinematic region for reasons that I'll point out later.

I'll describe the motivation for these experiments and their execution with particular emphasis on the role that Kent played. In doing so I will try to convey to those who were not active in this field during this period, some of the flavor of the ideas behind the experiments and techniques used to do them. My presentation will follow pretty much in the historical order in which events took place. 


\section{RHO PRODUCTION PROPOSAL}

\section{A. Motivation}

My story begins in 1965 with a proposal to the ZGS at Argonne National Lab to study the $\rho$ production and decay reactions

$$
\begin{aligned}
\pi^{-} p & \rightarrow \rho^{0} n, \quad \rho^{0} \rightarrow \pi^{+} \pi^{-} \\
& \rightarrow \rho^{-} p, \quad \rho^{-} \rightarrow \pi^{-} \pi^{0} .
\end{aligned}
$$

I'll describe our interest in these reactions and our method of studying them in some detail. Even though my main focus will be on backward production of $N^{*}$ s, the $\rho$ experiment came first and fixed the apparatus that was available for the $N^{*}$ experiment.

We were interested in the $\rho$ reactions because experience in the early 60's had shown that it might be possible to model them in a fairly principled way. There were two features of observed reactions that led to this hope.

1) A significant fraction of multi particle production takes place through quasi two-body states, or resonances, as illustrated schematically in Fig. 1 for the special case of $\rho$ production. There, a stable nucleon is produced together with an almost stable $\rho$ having a fairly well defined mass; the resonant $\rho$ then decays into two stable pions leading to a three particle final state.

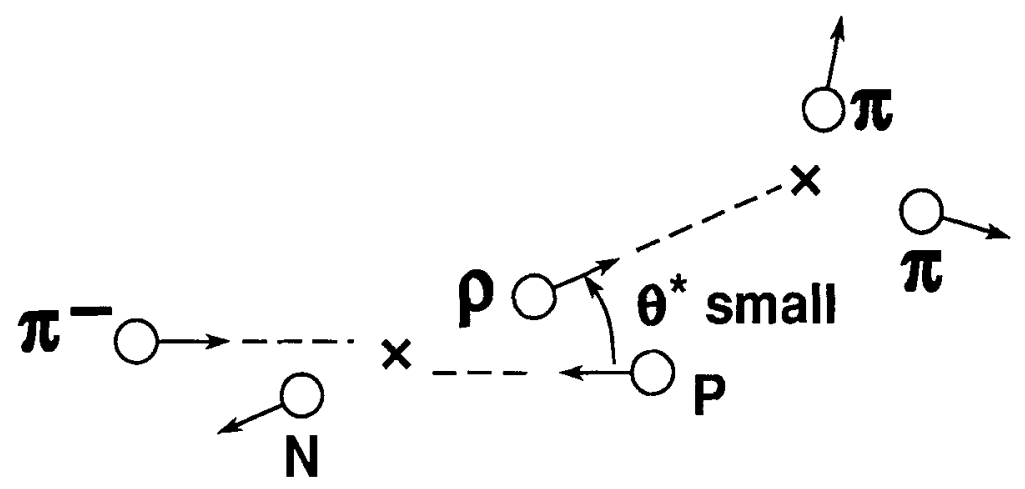

Fig. 1. Schematic diagram of the peripheral production and subsequent decay of a $\rho$ meson as seen in the production C.M. frame. 
2) The resonant particles are produced nearly in the forward (or backward) direction in the center of mass frame as indicated by $\cos \theta^{*} \simeq 1$ in Fig. 1 .

This means that the reacting particles receive only a very small transverse momentum kick during their production and that they have been produced in a grazing, or peripheral, collision. In the context of

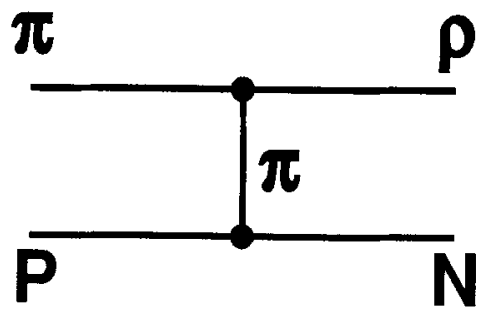
quantum field theory, such a process would correspond to the exchange of a light particle as diagramed in Fig. 2 for $\rho$ production.

The pion is the natural candidate as the mediator of $\rho$ production since it is the lightest strongly interacting particle and it satisfies the necessary conservation laws at both vertices. Models involving pion exchange were called OPE for one pion exchange. In these models the square of the four- momentum transfer, $\Delta^{2}=\left(p_{\rho}-p_{\pi}\right)^{2}$, from the $\pi$ to the $\rho$ is used to specify the production scattering angle.

Examples of the evidence leading to the OPE model are shown in Fig. 3 on the next page. Fig. 3a shows the distribution in mass of the $\pi^{+}, \pi^{0}$ produced in the reaction $\pi^{+} p \rightarrow \pi^{+} \pi^{0} p$ at an average beam momentum of $2.6 \mathrm{GeV}$. In this case, about 40 percent of the final state goes through the $\rho$ resonance. This result comes from a hydrogen bubble chamber experiment ${ }^{1}$ carried out at the Brookhaven AGS around 1960.

Fig. 3b is taken from a 1965 review article by J. D. Jackson. It shows the angular distribution of produced $\rho^{+}$from the same reaction compared with some theoretical predictions of the OPE model. The curves in this figure are comparisons of the OPE model with experiment and bring me to one of the major reasons for our interest in $\rho$ production. The straight field theory prediction of the OPE model is the top curve and is in gross disagreement with the data. The lower curves are modifications of the field theory result that were expected to be reasonable based on then current understanding of strong interactions. The solid curve is a prediction of a modification to OPE called the absorption model and was the main point of Jackson's review article. This model 


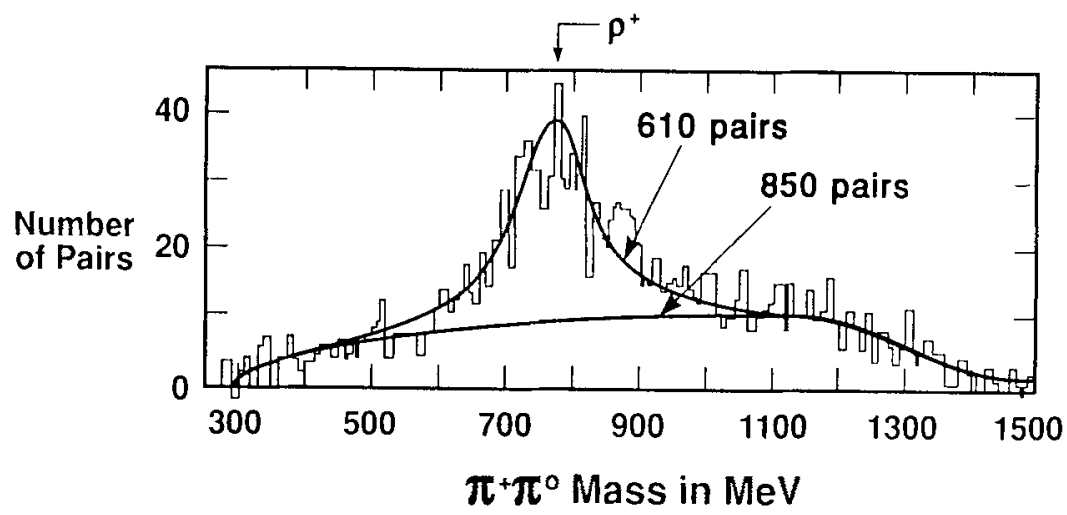

(a) $\pi^{+} \pi^{0}$ mass distribution from $\pi^{+} p \rightarrow \pi^{+} \pi^{0} p$ at $2.6 \mathrm{GeV}$.

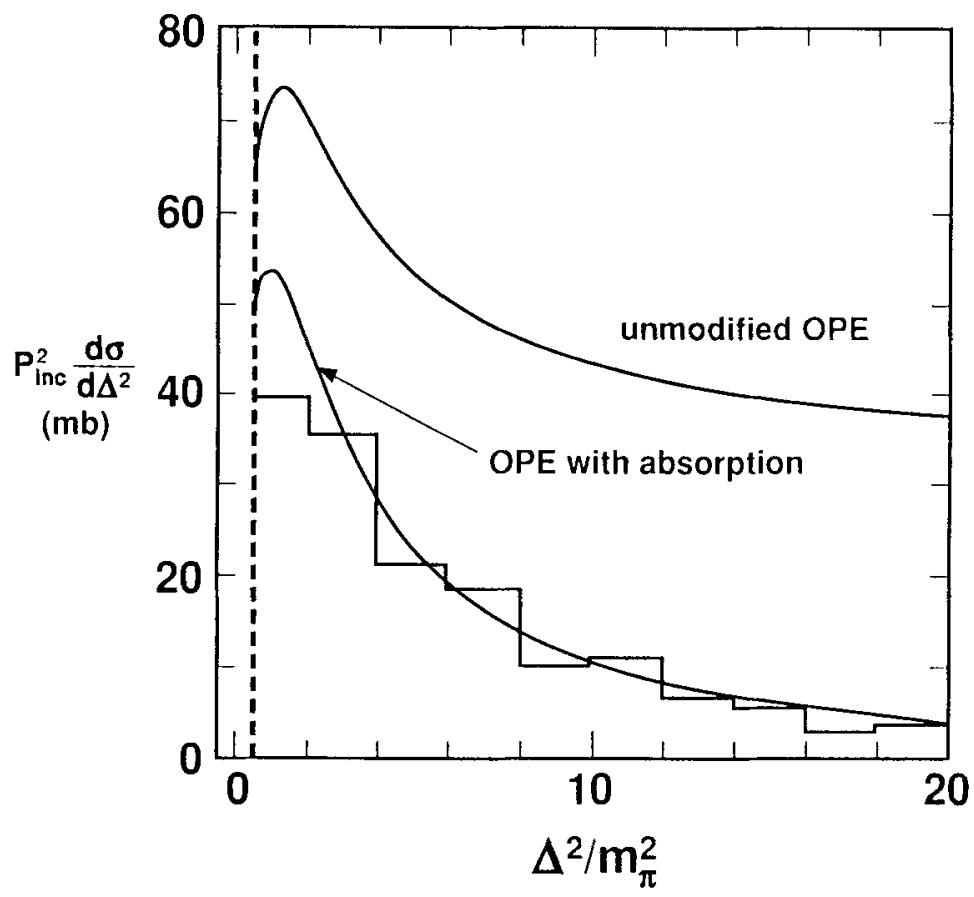

(b) $\rho^{+}$production angular distribution for $\pi^{+} p \rightarrow \rho^{+} p$.

Fig. 3. Illustrating evidence for the OPE model. (a) Distribution in $\pi^{+} \pi^{0}$ mass at $2.6 \mathrm{Gev}$ beam momentum from Ref. 1 . (b) Production center of mass distribution of $\rho^{+}$from Ref. 2. The histogram is data and the solid curves are unmodified and absorption modified OPE predictions. 
recognized that reactions not involving resonance production may be relatively violent ones occurring at small impact parameter. These processes can use up part of the flux at small impact parameter before resonance production can take place. The absorption model altered the low impact parameter part of the OPE amplitude to account for this flux loss, and in doing so, produced a sharper and smaller production differential cross section that could account for the data. I might mention at this point that the absorption model was a natural topic of interest to experimenters at Michigan because some of our theorists, particularly Marc Ross, and later Gordon Kane, had done some of the original work on the subject.

The differential cross section of Fig. $3 \mathrm{~b}$ does not provide a very sensitive test of a particle exchange model. More sensitive are the details of the angular distribution of the products of the decaying resonance. The study of such a decay distribution takes a very large amount of data, much more than typical bubble chambers experiments had been producing at that time. It was our hope that by making use of the triggerable feature of the spark chamber, we could collect a data sample that was much richer in $\rho$ events than the samples collected with bubble chambers ( at that time, triggered bubble chambers were not yet in use ). These were the considerations that led us to propose the $\rho$ experiment in 1965.

B. Trigger Scheme and Apparatus

In order acheive the desired $\rho$ enrichment, we needed some special feature of the $\rho$ production process that would distinguish it from the bulk of interactions, and could then be the basis for a trigger. For the nearly forward $\rho$ 's of interest for the OPE model this feature was that the recoiling nucleon emerged from the interaction almost sideways from the projectile direction and moving slowly as shown schematically below in Fig. 4.

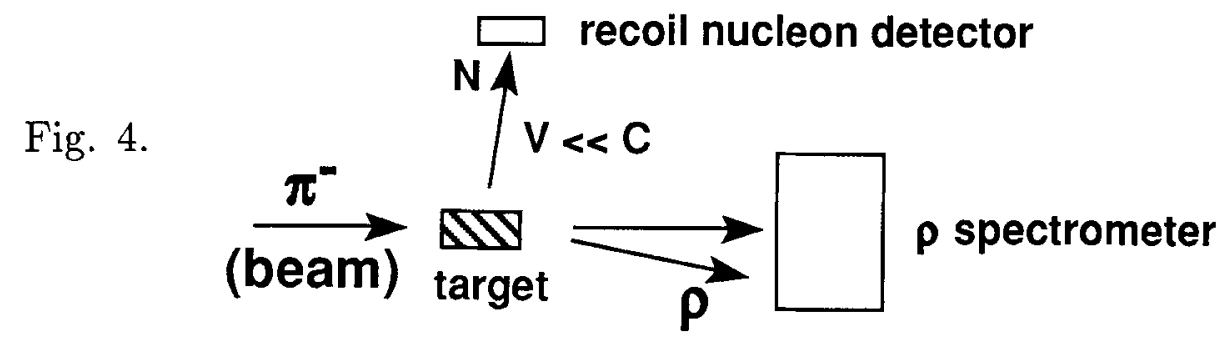


Since such a slow nucleon would move at only a few tenths of the speed of light, a delayed coincidence timed on these nucleons would discriminate against the bulk of $\pi p$ interactions which would send faster particles toward the nucleon detector. A forward spectrometer to measure the $\rho$ decay products completed the essential elements in the design as illustrated in Fig. 4.

The kinematic range to be explored in this experiment can be seen in Fig. 5, where the lab angle of the recoiling nucleon is plotted as a function of of its kinetic energy for various values of the dipion mass. The horizontal lines are the angular limits placed by our nucleon detector and the vertical lower limit on kinetic energy was set by the requirement that the nucleon have sufficient energy to trigger the detector. The point of the graph is that most of the $\rho$ action in the one pion exchange model takes place at $\Delta^{2}$ less than $0.4 G e V^{2}\left(\Delta^{2} / m_{\pi}^{2}<20\right.$ in Fig. 3b ), and in this kinematic region the recoiling nucleon could be detected in an essentially unbiased way.

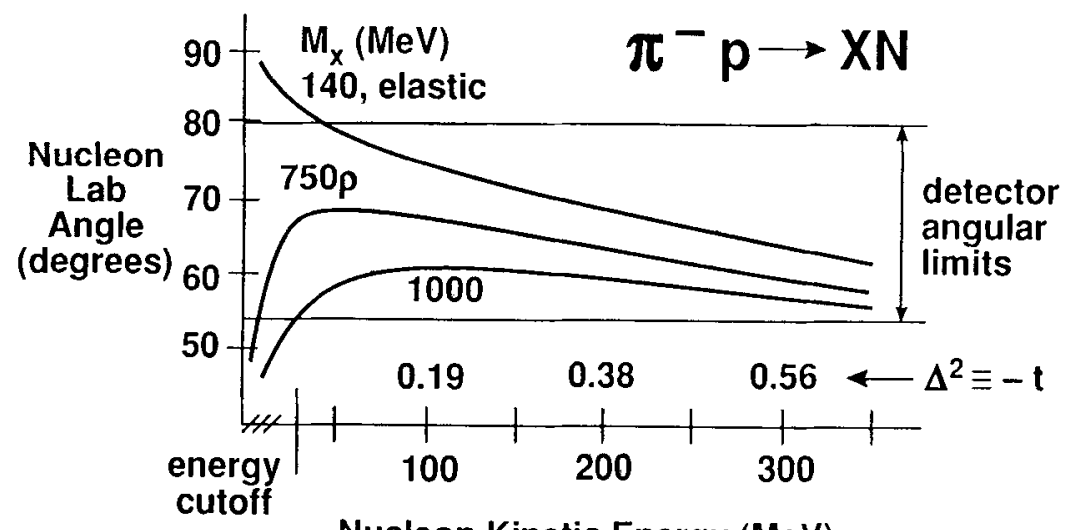

Nucleon Kinetic Energy (MeV)

Fig. 5. Diagram illustrating that the recoiling nucleon has small momentum transfer, and is emitted almost sideways to the beam direction, over a wide range of dipion mass, $M_{x}$.

Our proposed setup exploiting this trigger is shown in Fig. 6 on the next page. Recoiling slow neutrons were to be detected by a large block of plastic scintillator, $\mathrm{N}$, in veto with a thin scintillator, $\mathrm{P}$, in front of it. Protons were to be detected as P OR N. The overall trigger was either a proton or neutron signal in delayed coincidence with an interacting beam particle. 
The remaining features of the experiment can also be seen from Fig. 6. Arrays of optical spark chambers would determine charged pion directions for most possible decay configurations of the rho. In addition, a large aperture magnet would give the momentum of a charged pion often enough to permit constrained fits for most of the $\rho$ events. For the $\rho^{-} \rightarrow \pi^{+} \pi^{0}$ part of the experiment, a lead plate to convert the $\pi^{0}$ decay gammas would be used to give sufficient information for a constrained fit when the $\pi^{-}$did not go through the magnet.

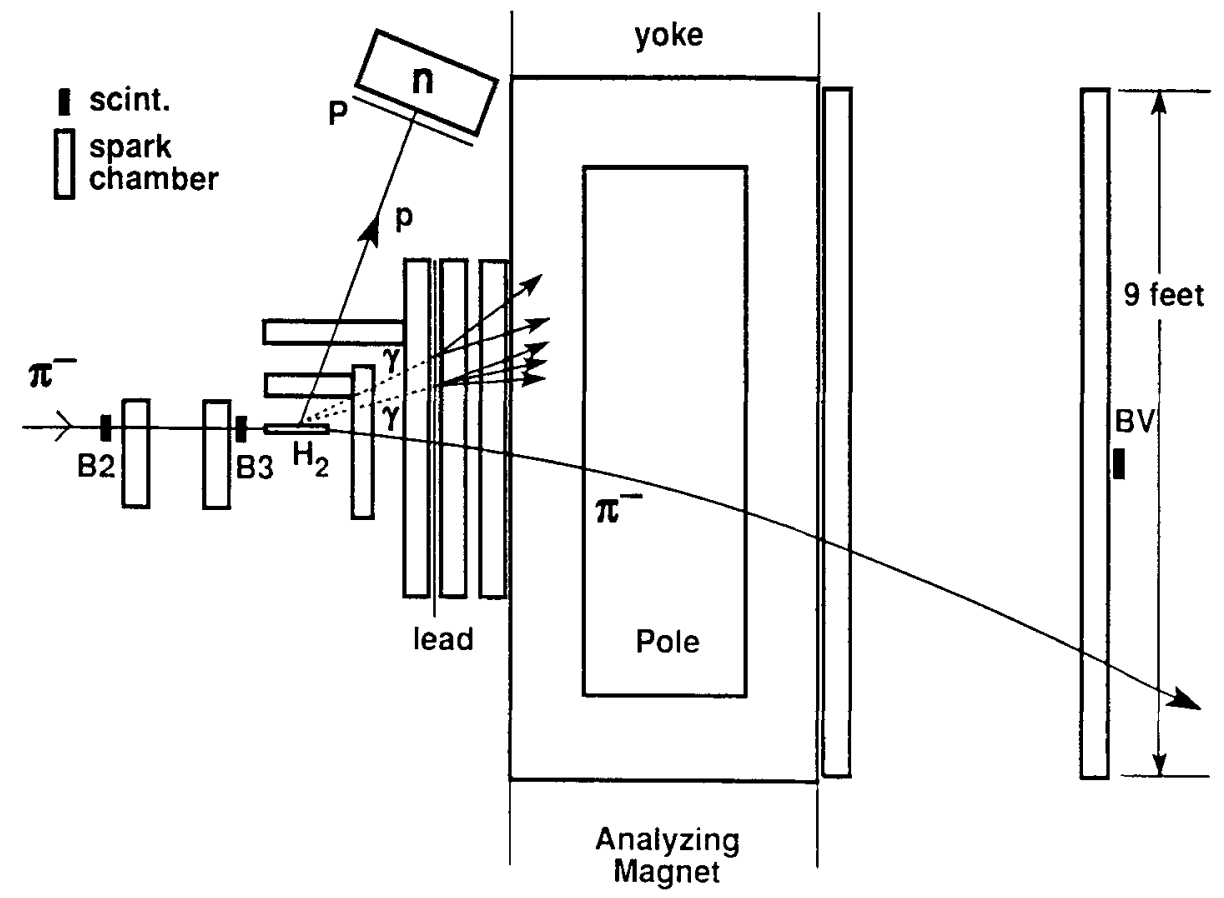

Fig. 6. Plan view of the apparatus proposed for study of forward production and subsequent decay of $\rho^{\prime}$ s in $\pi^{-} p$ interactions.

This then was our proposed method for studying $\rho$ production and is pretty much what we eventually did. For the purposes of this discussion, I should emphasize the scale of size of the apparatus shown if Fig. 6 . The analyzing magnet was 16 feet wide and 4 feet long in the beam direction. The large spark chambers upstream of the magnet were 4 feet square while those downstream were 8 feet wide by 3 feet high. These were very large chambers compared with our earlier ones, and compared with those of most 
other experiments at the time. This change in scale caused us all kinds of problems as I will explain in a moment.

The proposal was for the $17^{0}$ beam at the ZGS with a beam momentum of $5 \mathrm{GeV}$. High beam momentum was good because the increased forward folding of $\rho$ decay products in the lab gave us more charged particles through the analyzing magnet and because one pion exchange models were presumably more valid at high momentum. $5 \mathrm{GeV}$ was a compromise between these considerations and not being hurt too much by the decrease in beam flux as momentum increased.

\section{PREPARING FOR THE $\rho$ EXPERIMENT}

We began to build the apparatus at the beginning of ' 65 at the same time that we submitted our proposal. The cast of characters working on this experiment at the start consisted of - Tris Coffin, Don Meyer and Kent Terwilliger ( faculty); Noel Stanton ( post doc ); and Bob Anthony, Ed Meanley and Jim Rice (graduate students ).

Most of our activity during the first year and a half of preparation was devoted to building the large spark chambers and making them work. Fig. 7 on the next page shows the chamber construction. The active elements were one mil aluminum foil stretched flat on aluminum frames. These frames were supported by slots machined in plexiglas frames. The chambers were activated by applying a pulse of about 10 kilovolts to alternate plates when a trigger signalled that an interaction of interest had taken place. This ideally leads to formation of a nice fat spark along the path of a charged particle as indicated in Fig. 7. We very quickly encountered problems that had not been present in our previous experiments with small spark chambers.

The worst of these problems was the presence of reflections of spark light from the plexiglas frame on the side of the chambers farthest from the camera ( the bottom side in Fig. 7 ). These reflections were initially almost as bright as the spark itself and absolutely had to be eliminated before the chambers could be used in an experiment. We tried painting the rear plexiglas surfaces with a variety of black paints, but all of them caused sufficient sparking to occur along the painted surface that the chambers did not work properly. We eventually found a Dow Corning silicone rubber preparation that could be tinted dark and coated on plexiglas without causing breakdown. Unfortunately, this material was still shiny enough when it dried to cause reflections. It was then 


\section{Lucite wall window and frame support}

\section{to camera}

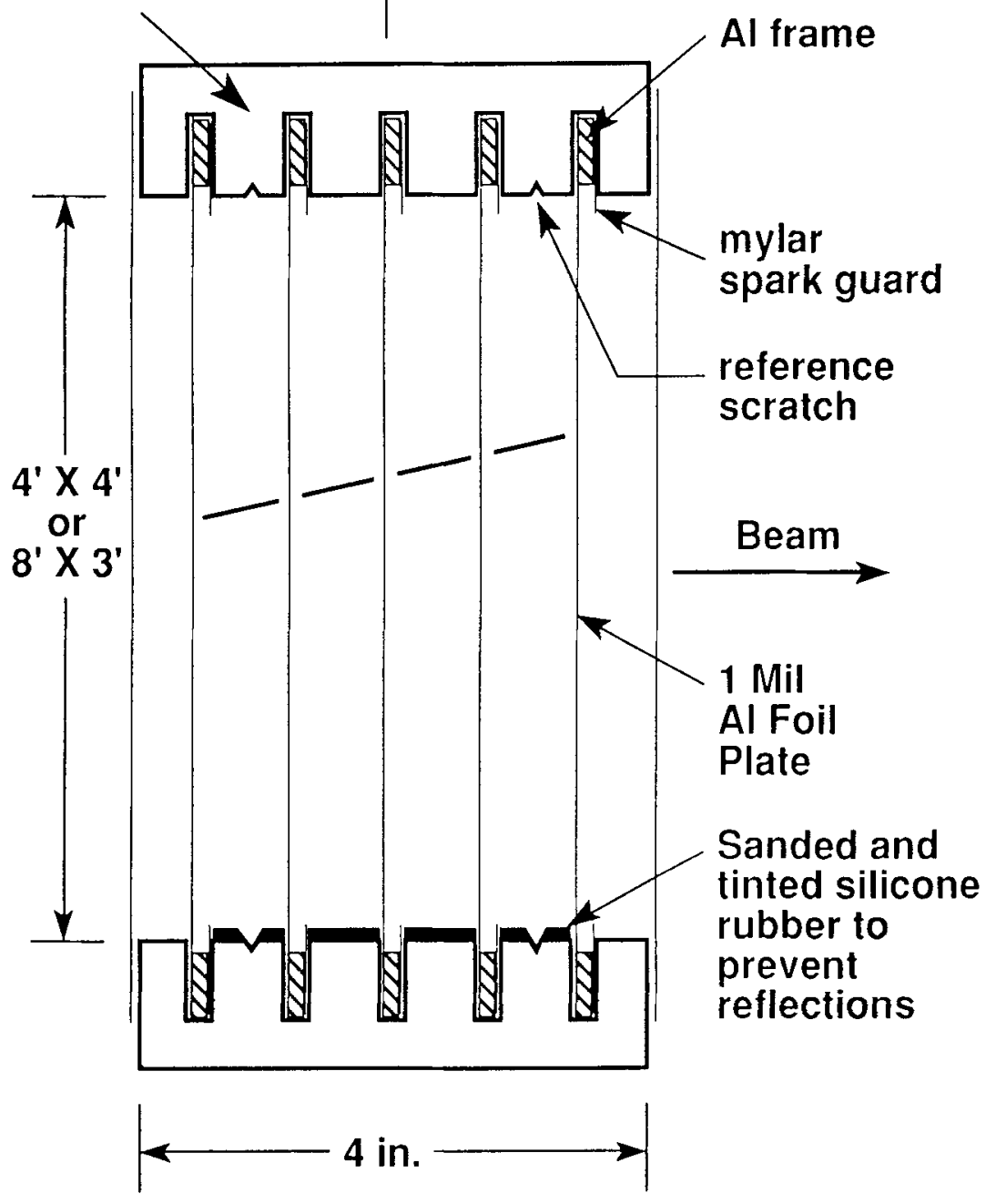

Fig. 7. Side view of a spark chamber. Sanded and tinted silicone rubber on the bottom frame was necessary to eliminate reflected spark light. Illuminated reference scratches permitted an accurate real to film space transformation in spite of large mirror distortions. 
necessary to hand sand all of the silliconed surfaces to dull them enough that they could not reflect. As I remember, it took about a year to solve this problem. Fig. 7 also shows an end view of sets of scratches cut into the plexiglas frames. These were needed to solve another problem associated with large chambers as I now describe.

Our spark chamber data was read out by using mirrors to reflect plan views and two side views of the chambers onto a single frame of $35 \mathrm{~mm}$ film as shown schematically in Fig. 8a. We had planned to relate real space to film space by including a small number of square luminescent fiducial panels of known position in each view. We discovered however that we could not keep our large mirrors flat, so that serious optical distortions were introduced into the relation between real space and film space. Since we already had the mirrors and could not afford to buy optically flat ones, we had to improvise a correction scheme. In order to correct for the distortions we installed a very extensive set of grid marks on all of the chamber faces. Fig. 8b shows one set of scratch marks for the top view and one of the side views of a chamber. We had to periodically illuminate these scratch marks and photograph them during the experiment to work out the transformation from film to real space with sufficient accuracy. Unfortunately, we did not know that this extensive set of scratches was needed when we were first building the chambers, so it was necessary to take them apart, put the scratches in, and then reassemble them.

There were other more minor problems that were new to us and that had to be solved during this period. Proper firing of the large chambers required much more charge delivered at high voltage than had been needed in our earlier experiments. This required the development of spark gaps to act as a high current switch. This was also our first experience with having to correlate a large amount of analog information, particularly times of flight, with spark chamber records that were on film. This was done by converting analog signals to binary form and than lighting luminescent panels that would be photographed with the chamber pictures. I remember being very relieved when Noel stanton joined us as a postdoc and took over the development of this system.

This brings me to the point in our preparations for the $\rho$ experiment where it had been approved and where the major components of the apparatus were under control. At about this time a new element was injected in our thinking when we realized that 


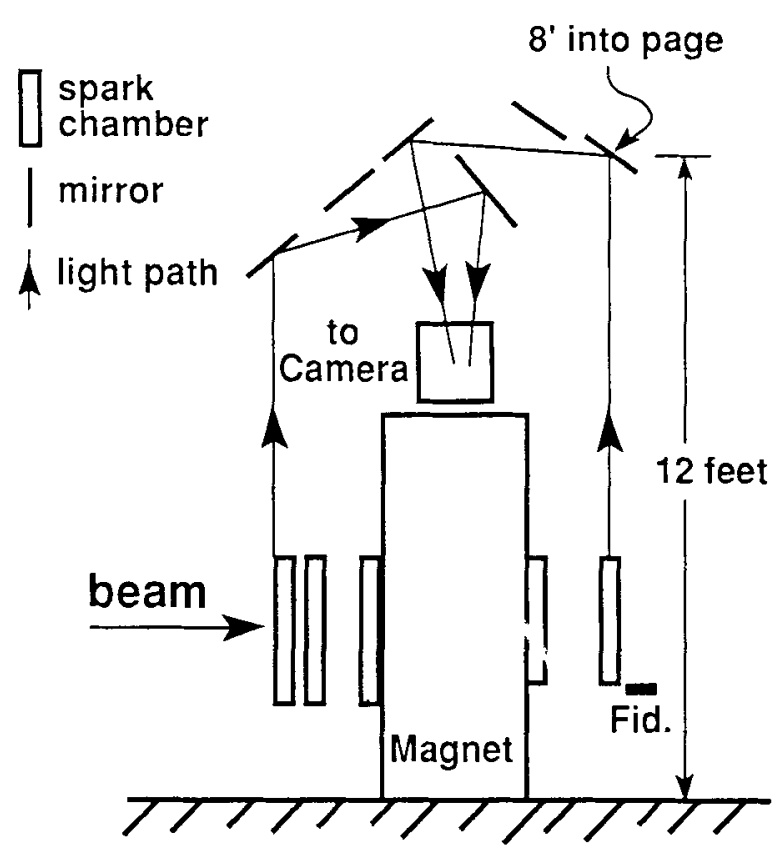

Fig. 8a

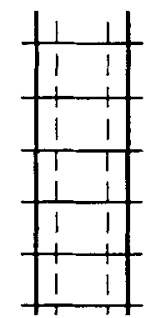

SIDE
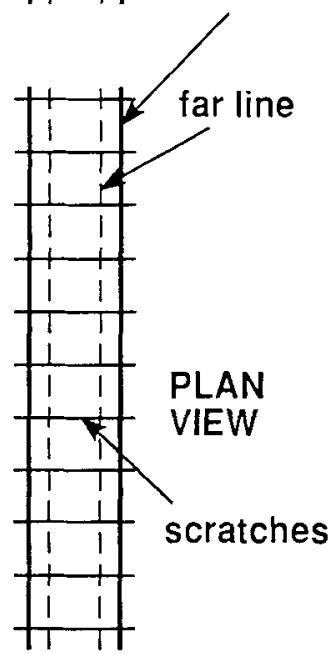

Fig. $8 b$

Fig. 8. a) Elevation view illustrating the optical system. Note that the top mirrors were $8 \mathrm{ft}$. long into the page. b) Reference scratches in film space. 
it would be possible to do another quite different experiment with the same apparatus; this was the backward $N^{*}$ experiment. Pressure to have such an alternative experiment had been building ever since Kent's return from sabbatical about six months earlier. In order to explain this pressure I will outline the historical sequence of events leading up to the $N^{*}$ proposal.

1 July 1964

2 Sept. 1964

3 Jan. 1965

4 July 1965

5 Sept. 1965

6 Sept. 1865

7 Feb. 1966

8 June 1966

9 July 1966 $\rho$ experiment conceived.

Kent left for a CERN sabbatical.

$\rho$ proposal submitted to ZGS.

Kent returned from sabbatical.

Don Meyer left for Cern sabbatical.

Noel Stanton joined group as a postdoc. $\rho$ experiment approved and scheduled for fall 1966.

Major chamber problems solved.

Backward $N^{*}$ experiment conceived, proposed and approved.

There are two related points that can be seen from this outline. The first is that even though Kent had been a party to the initial $\rho$ proposal, he was away on sabbatical during much of the detailed planning and construction. The second point is that the backward $N^{*}$ experiment was conceived, proposed and approved within a period of a month. This came about as follows.

When Kent returned from CERN, he soon made it clear that he had lost some of his enthusiasm for the $\rho$ experiment. He was afraid that the experiment might not work, and that even if it did, it might not be as significant as we had hoped. I recall that he was particularly worried that spurious triggers from effects such as accidental coincidences between nucleon detector and beam halo might swamp the real events. This kind of effect is very difficult to estimate; you almost have to be in place in a beam to find out how bad it is. In any case, it was clear that Kent was thinking about alternatives to the $\rho$ experiment throughout the winter and spring of 1966 while we were trying to get the the $\rho$ apparatus to work. I don't remember any specific possible alternatives being mentioned until late June of 1966, when one appeared during a train ride from Ann Arbor to Chicago. 


\section{BACKWARD $N^{*}$ PROPOSAL}

\section{A. History}

In the 60's the best way to get from Ann Arbor to Argonne National Lab was by New York Central Train. You got the train right in Ann Arbor, had a nice dinner during the ride, and were met at Chicago's Lasalle Street station by a very respectful and deferential limousine driver who whisked you down to Argonne in about a half hour. The driver also entertained you by bringing you up to date on the latest gossip about other experimenters.

During such a trip to Argonne in early July, Kent asked me if I had any experiments in mind that I would like to do after the $\rho$ experiment. I mentioned that I had been interested for some time in backward scattering of protons, and possible backward production of nucleon resonances. In the center of mass frame of a $\pi^{-} p$ interaction, this would appear schematically as in Fig. 9 below.

Fig. 9. Center of mass view of backward $N^{*}$ production.

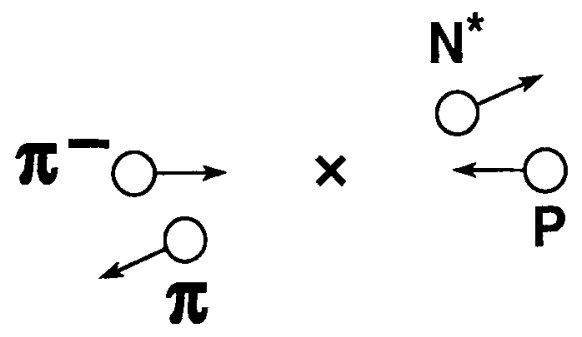

For reasons that I'll mention later, it seemed that this would be an interesting process to study, and that the kinematics were such that we could study it with essentially the same apparatus that we had built for the $\rho$ experiment. Kent responded to this by saying that he had been thinking exactly the same thing. He then suggested that we propose it right away as an experiment to follow the $\rho$ experiment.

I remember this experience very vividly for several reasons. First, I was surprised that even though we were thinking along very similar lines, the subject had not come up sooner. The reason for this is probably that we had both been so concerned about our chamber problems that we weren't sure that we would be able to do any experiment at all. Having finally solved most of these problems, we felt free to think about the future. The second reason for my remembering this episode so well it that I was amazed by Kent's energy, and what seemed to me to be audacity. I still thought that the $\rho$ experiment would work, but that it would be very demanding and would be all that we could handle in the 
immediate future. I had pictured a second experiment as taking place after some respectable interval, such as a year. I was somewhat appalled by the thought of running two long experiments right in a row.

In any case, by the time we reached Chicago, Kent had sketched out a proposal for the backward $N^{*}$ experiment, and I had recovered enough to go along with it. It is my recollection that Kent asked for and was granted a meeting with the ZGS program committee just a few days later, and that the experiment was approved several weeks after that. I believe that this was somewhat of a record for ZGS proposals.

This was a turning point in our approach to this set of experiments. Even though we subsequently ran the $\rho$ experiment before the $N^{*}$ one, we analyzed the $N^{*}$ experiment first and generally gave it the most attention. Most of the rest of my remarks will concern this $N^{*}$ experiment. I will briefly discuss our reasons for suggesting it, the experimental run, analysis of data, and what we learned from it. I will make one comment on the $\rho$ experiment at the end.

\section{B. Motivation for the $N^{*}$ Experiment}

There had been several experimental discoveries in the few years preceding our proposal that suggested that backward peaks might exist as a general feature of strong reactions. One of these discoveries is illustrated in Fig. 10 ( next page), which shows differential cross sections for several of the final states in $\pi^{+} p$ reactions at $4 \mathrm{GeV}$. This data came from an experiment by a large European bubble chamber collaboration published ${ }^{3}$ in 1965 . For all of the states listed in Fig. 10, there are large forward peaks and small backward peaks.

There was quite a bit information on backward elastic scattering from several experiments carried out at the ZGS by Michigan groups. ${ }^{4,5}$ Fig. 11 (two pages forward ) shows a combined energy and angle distribution for $\pi^{-} p$ elastic scattering near the backward direction. This data showed large energy and angle fluctuations at low momenta near $2 \mathrm{GeV}$, but seemed to suggest the onset of a sharp backward peak that varied smoothly with energy as energy increased. Several isolated measurements from higher energy CERN and Brookhaven experiments were consistent with this view.

In addition, there was a growing suspicion that the backward peak might be associated with baryon exchange. Barger and Cline ${ }^{6}$ 

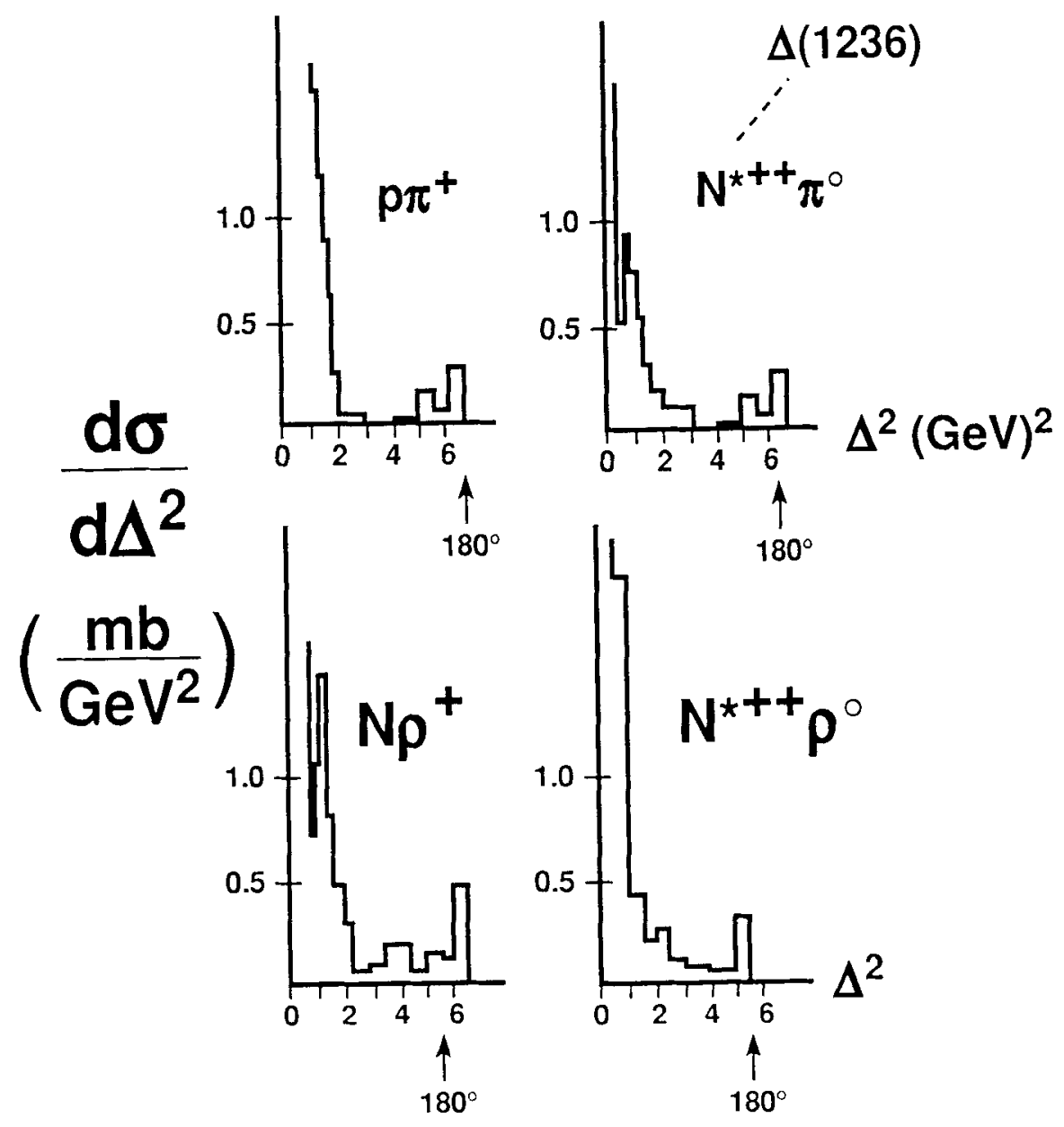

Fig. 10. Illustration of the presence of forward and backward peaks in $\pi^{+} p$ interactions at a beam momentum of $4 \mathrm{GeV}$. The final state described by each differential cross section is identified by the symbols on the diagram. These graphs are taken from reference 3 . 


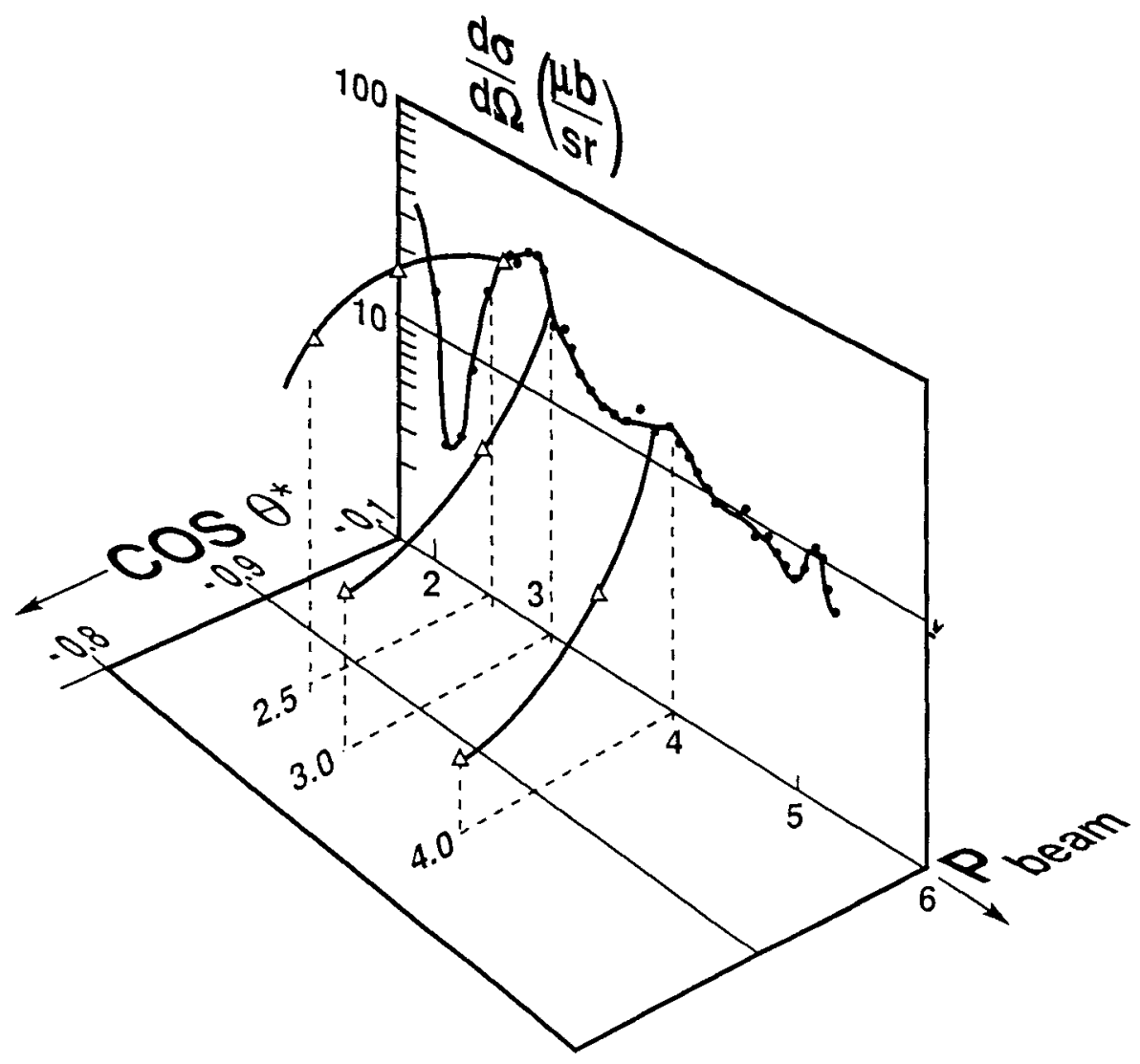

Fig. 11. Summary of the dependence of the $\pi^{-} p$ backward elastic scattering differential cross section $\frac{d \sigma}{d \Omega}$ on center of mass angle $\theta^{*}$ and on beam momentum $p_{\text {beam }}$. The points are from reference 4 and the triangles are from reference 5 . 
had recently interpreted the energy dependence of the backward data of Kormanyos et.al. ${ }^{4}$ as being due to the interference between an amplitude due to direct channel resonances and a baryon exchange amplitude described by Regge theory.

It was clear that more angular distributions in elastic scattering would be useful, and we wanted to study this, but we were most interested in looking for backward production of excited nucleon states. Our proposal was to explore the backward direction at a number of momenta between 2 and $6 \mathrm{GeV}$.

C. Trigger Scheme and Apparatus

We proposed to study the specific processes

$$
\begin{aligned}
\pi^{-} p & \rightarrow p \pi^{-} \\
& \rightarrow N^{*+} \pi^{-} \\
& \rightarrow N^{*-} \pi^{+},
\end{aligned}
$$

with the apparatus of Fig. 12 shown on the next page. The kinematics are very simple here. Since the projectile pion is lighter than the target proton, it will bounce backward in a head-on elastic collision, as a golf ball bounces back from a bowling ball. It turns out that the back scattered pion has a momentum of about 400 $\mathrm{MeV}$, almost independent of the incoming beam momentum. As $N^{*}$ mass increases, more and more kinetic energy is converted to mass and the backward pion momentum decreases. If the magnet is tuned to give good momentum resolution on a $400 \mathrm{MeV}$ pion, then momenta below about $200 \mathrm{MeV}$ are not observable because they bend away too much. This translates into an $N^{*}$ mass range of about .94 to $2 \mathrm{GeV}$.

The trigger is also simple. A coincidence signal indicating an incoming beam pion is delayed and put in coincidence with a signal indicating a backward moving pion. As I have already suggested, most of the apparatus needed here was the same as that required for the $\rho$ experiment.

This is essentially the proposal that Kent submitted to the ZGS program committee. I am indebted to Jim Rice for providing me with a copy of the cover letter that Kent sent with the proposal. I include it below to illustrate his style. As you can see, it is brief, crisp and clear, all general characteristics of his writing. 


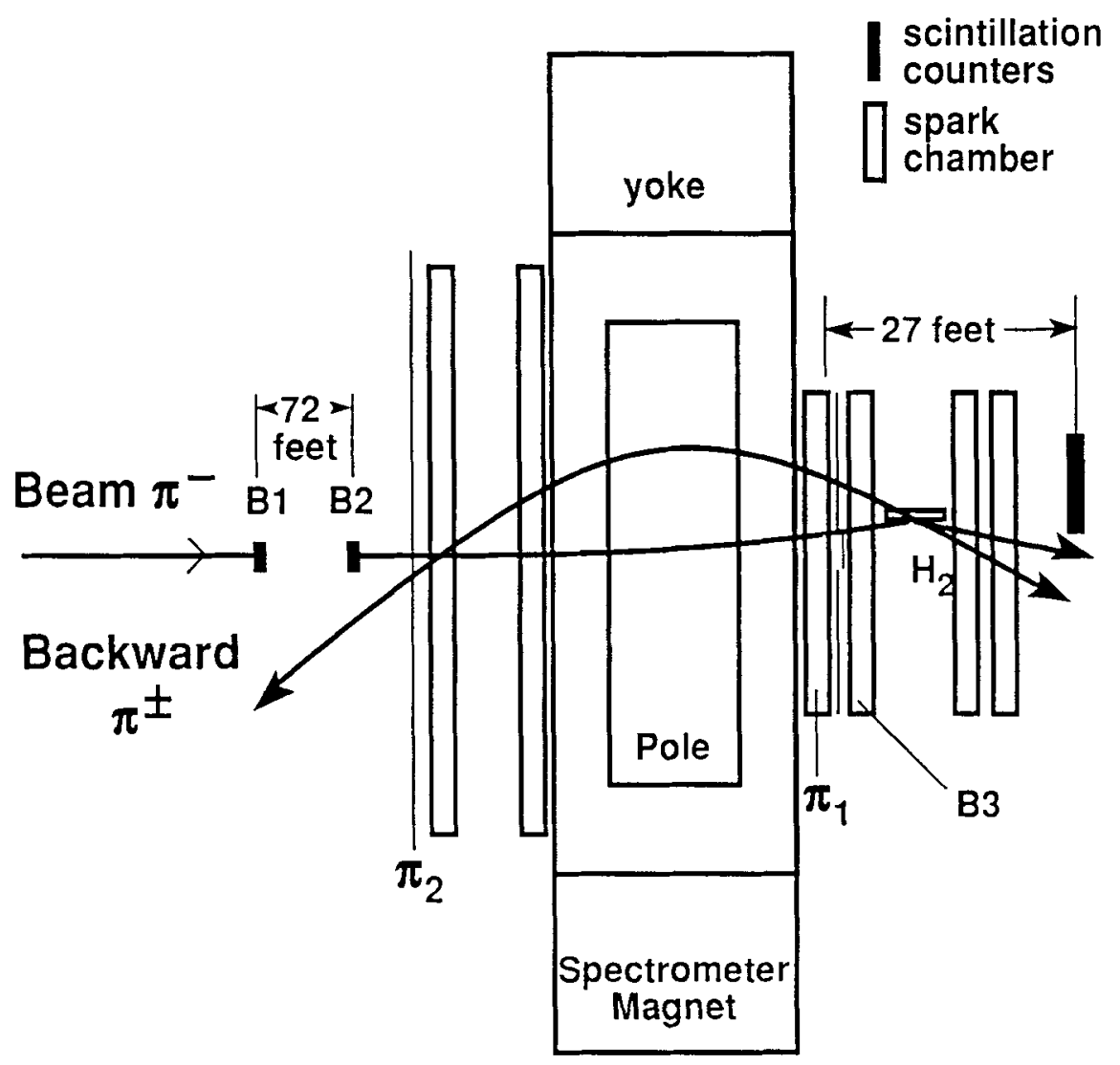

Fig. 12. Reconfiguration of the $\rho$ apparatus for the study of backward processes in $\pi^{-} p$ interactions. The drawing is to scale except for the beam veto, BV, which was $27 \mathrm{ft}$. downstream of the magnet to minimize vetoing events on $N^{*}$ decay products. 
The letter is addressed to Bob Sachs, the chairman of the ZGS program committee.

\section{Dear Bob}

Enclosed are copies of our proposal to study backward peaks in $\pi^{-} p$ inelastic and elastic scattering. We have previously discussed this experiment briefly with the program committee as an extension to our present E-40 experiment. You requested that since some heavy equipment moving was necessary, we submit it as a separate proposal. For this experiment, we require 45 shifts, (extendible to 60) and one week tune up time at low beam. Two weeks of changeover time should be adequate.

\section{RUNNING THE EXPERIMENTS}

We ran the $\rho$ experiment during the Fall of 1966, and the $N^{*}$ experiment during the winter of 1966 . I will make a few remarks about the run, and show some photos, to give the flavor of the times.

The experiment was located in the $17^{0}$ beam of the ZGS. This was a standard long term setup and is the same one that we used in our 1965 experiment. ${ }^{5}$ Fig. 13 on the next page shows the relationship between the ZGS ring, the beam magnets and our experiment. The scale of size is interesting. Even though it is miniscule by Fermilab standards, it nevertheless seemed big to us at the time. The main point to note about the beam for the purpose of my story is that Kent handled the beam tuning needed for our experiment. This was natural since he had a hand in the original design of the beam, and he was an expert on beam transport as you have seen in some of the other presentations. Our tuning turned out to be very tricky because of the nature of our target and reaction kinematics. For each of the 11 beam momenta in our experiment, it was necessary for Kent to spend several hours staring at monitor readouts and correction curves that he had prepared in advance. This was a highly intuitive process and could only be done quickly by someone with a lot of experience.

Photographs of the apparatus from several vantage points are displayed on the three pages following Fig. 13. Fig. 14 is the view from upstream looking at the most upstream ones of the backward pion scintillation counters. While this was logically a single counter, it was actually an OR of 14 separate large scintillators. The downstream end of the beam transport pipe is also clear in this view. 
144 Strong Interaction Experiments at the ZGS

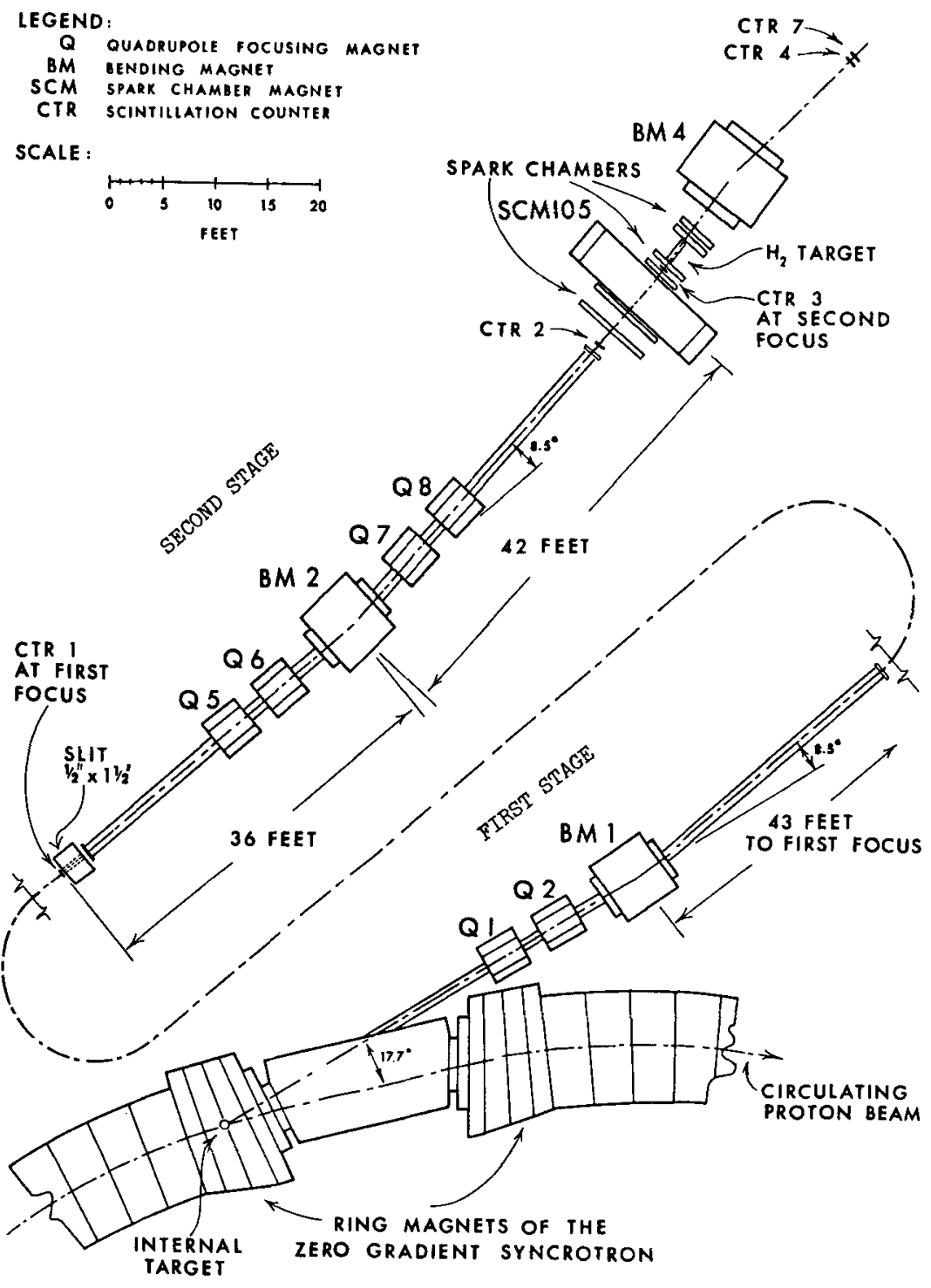

Fig. 13. The ZGS $17^{0}$ beam layout. Also shown are the beam defining counters, spark chambers and the SCM105 analyzing magnet used in our experiment. 


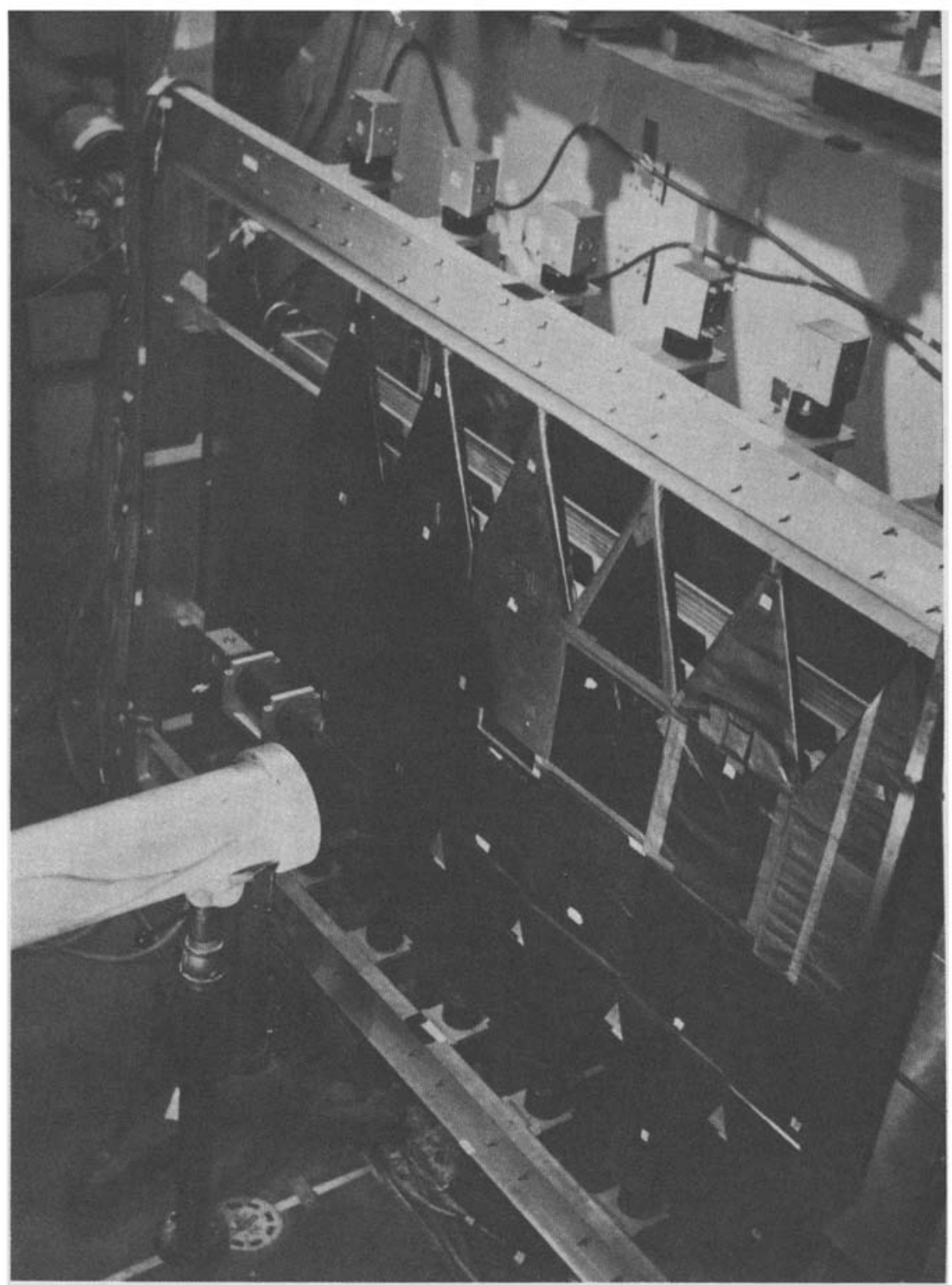

Fig. 14. Photograph of the $N^{*}$ apparatus from upstream of the SCM105 analyzing magnet showing the array of scintillation counters used in OR in the backscattered pion coincidence. The downstream end of the beam transport pipe is also visible. 


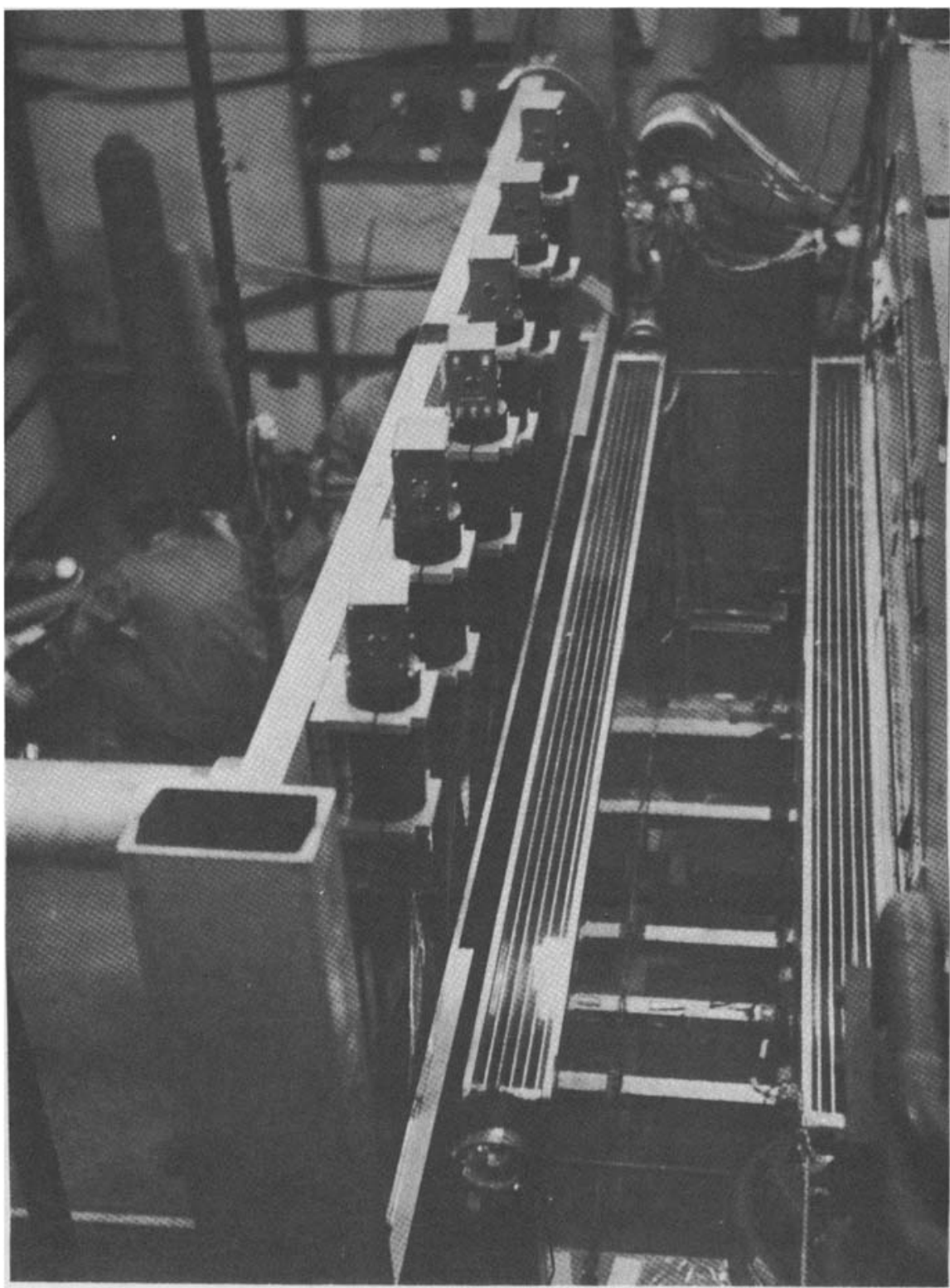

Fig. 15. View looking down on the apparatus from above the upstream end of the analyzing magnet. The large $3 \mathrm{ft}$. high by $8 \mathrm{ft}$. wide spark chambers are visible in this view. The author is squatting on the floor at left center. 


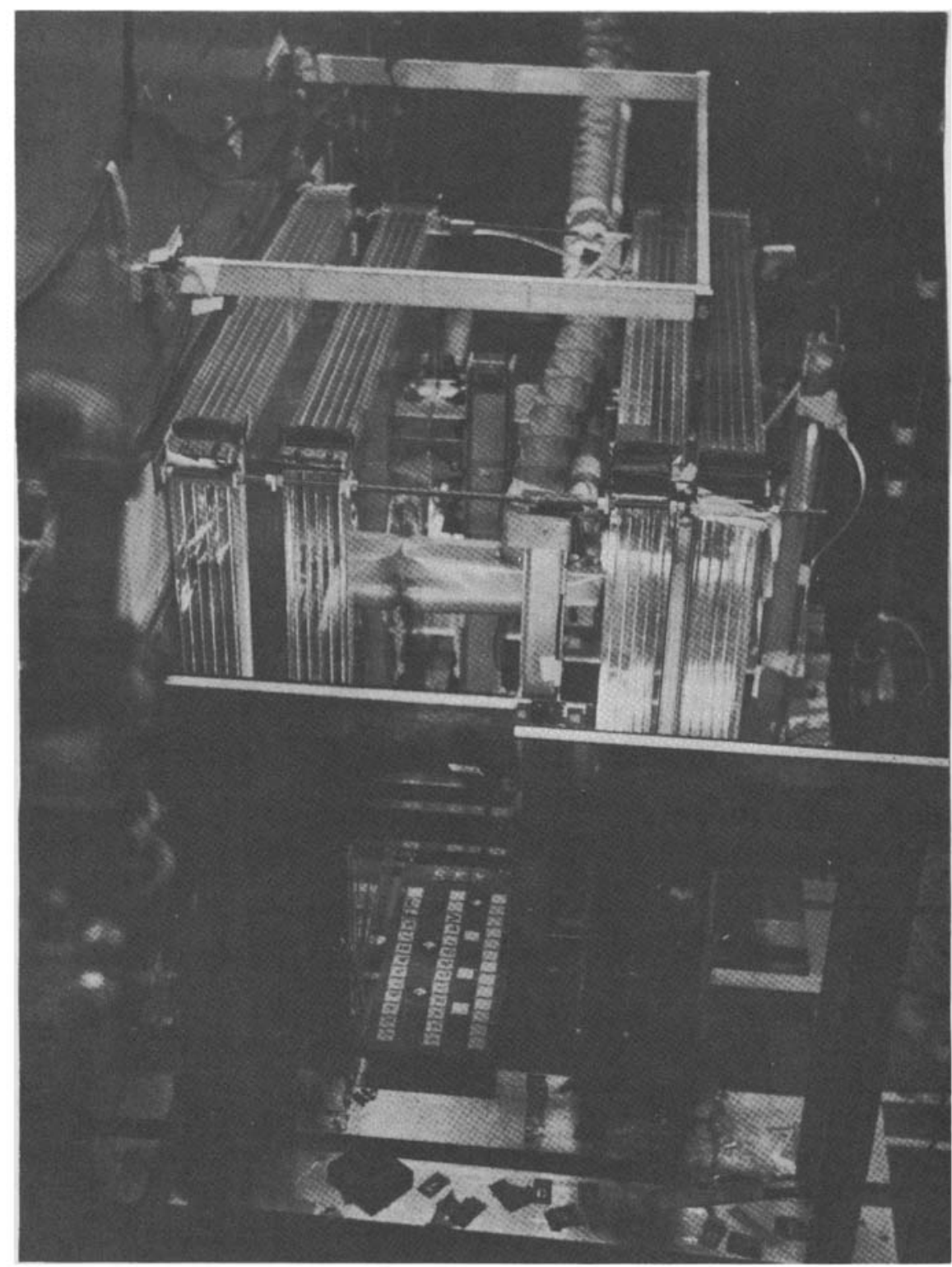

Fig. 16. View from above the downstream end of the analyzing magnet showing $4 \mathrm{ft}$. square spark chambers sandwiching the liquid hydrogen target ( bright streak in the center) and the luminescent panels used to record analog information in binary form. 


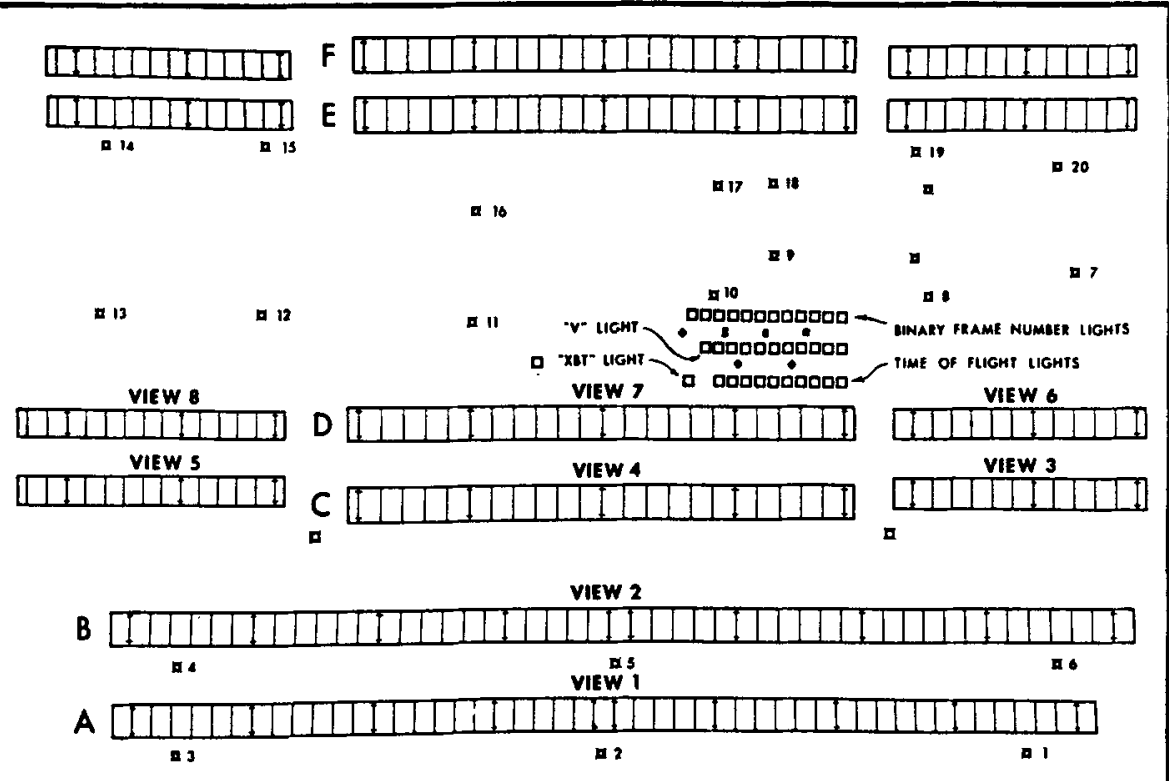

Figure 17.--Drawing of spark chambers, fiducials and data lights as they appear on film. Reference marks on the spark chambers are shown.

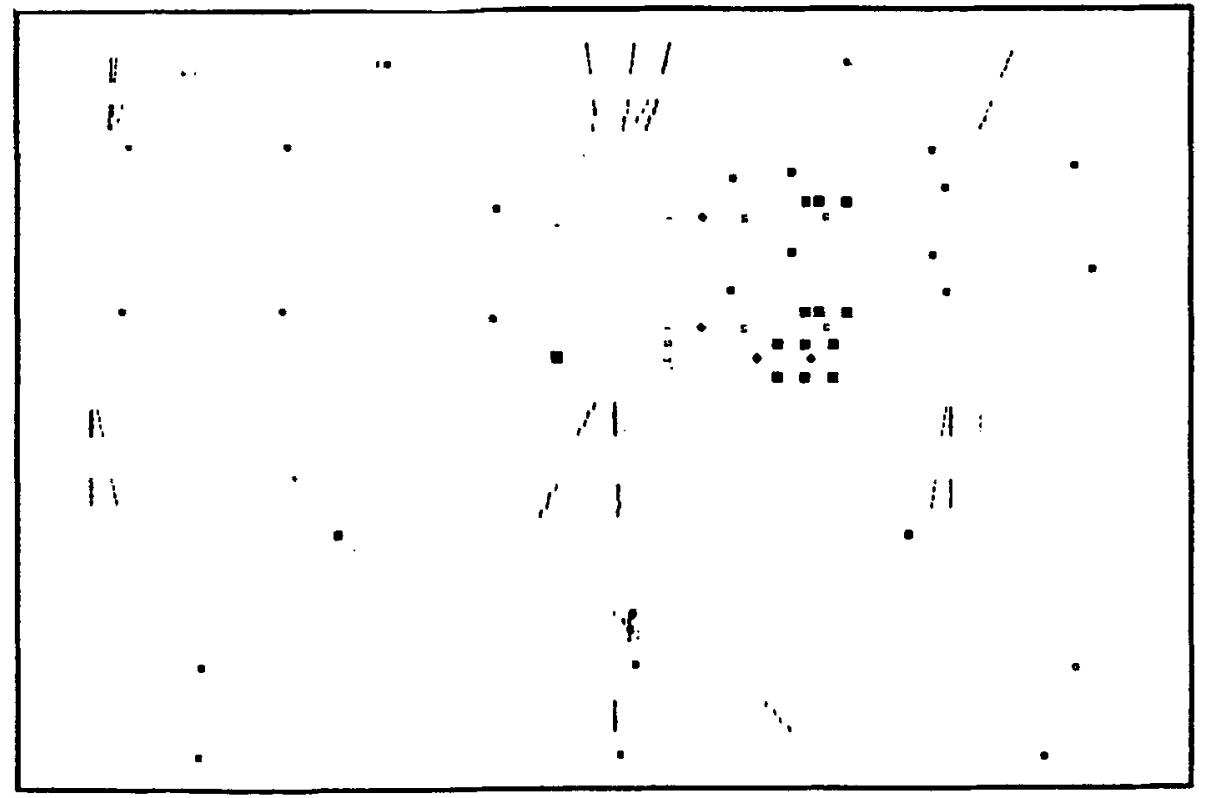

Figure 18.--Frame of film for a backward $\pi^{-} p$ scattering event. The objects in the photograph are spark chamber sparks, fiducial lights and data lights. 
Fig. 15 is a view looking down on the large 8 foot spark chambers that were just upstream of the analyzing magnet. The wall in the background was made of concrete blocks and served as a radiation shield. It was also a part of a light shield, together with an opaque plastic roof over the entire area shown, to keep room light out of the camera. The figure squatting in the foreground is the author, identifyable by the bald spot, probably in the act of stringing cables.

Fig. 16 shows the entire apparatus downstream of the magnet. The liquid hydrogen target is visible between the two sets of 4 foot square spark chambers. At the bottom are the luminescent panels used to transmit frame number and analog data such as the time of flight of the backward pion.

Finally, Figs. 17 and 18 on the preceding page show the layout of the chambers as they appeared on film, and a photo of a backward $N^{*}$ event on the same scale as the layout.

The completion dates of the $\rho$ and $N^{*}$ experiments are listed below together with the number of pictures taken in each.

\section{Rho completed Dec. 7, $1966 \quad 0.4 \times 10^{6} \mathrm{Pix}$ at $5 \mathrm{GeV}$. $N^{*} \quad$ completed March 6,1966 $1.7 \times 10^{6} \mathrm{Pix}$ at 11 beam momenta from $2-6 \mathrm{GeV}$.}

\section{DATA ANALYSIS}

I will comment on our data analysis only to the extent that it illustrates the essential role that Kent played in all parts of the experiment.

Since we had several million pictures to analyze we knew from the start that we would need to use an automatic scanning system, and we hoped that the one inherited from our earlier elastic scattering experiment would suffice. This turned out not to be the case, and the reason was again related to the much larger size of our chambers in the present experiments.

The essential elements of our autoscanning setup are shown in Fig. 19a on the next page. A CRT beam was stepped across the tube face under computer control. The CRT spot was focussed on the film so that its image stepped across the film. When a dark spark image was encountered, the beam was interrupted, and the spark coordinate recorded. In our earlier scattering experiment, a straight line scan of the CRT spot could be made to move right along the center of a spark chamber gap so that a very simple 
150 Strong Interaction Experiments at the ZGS

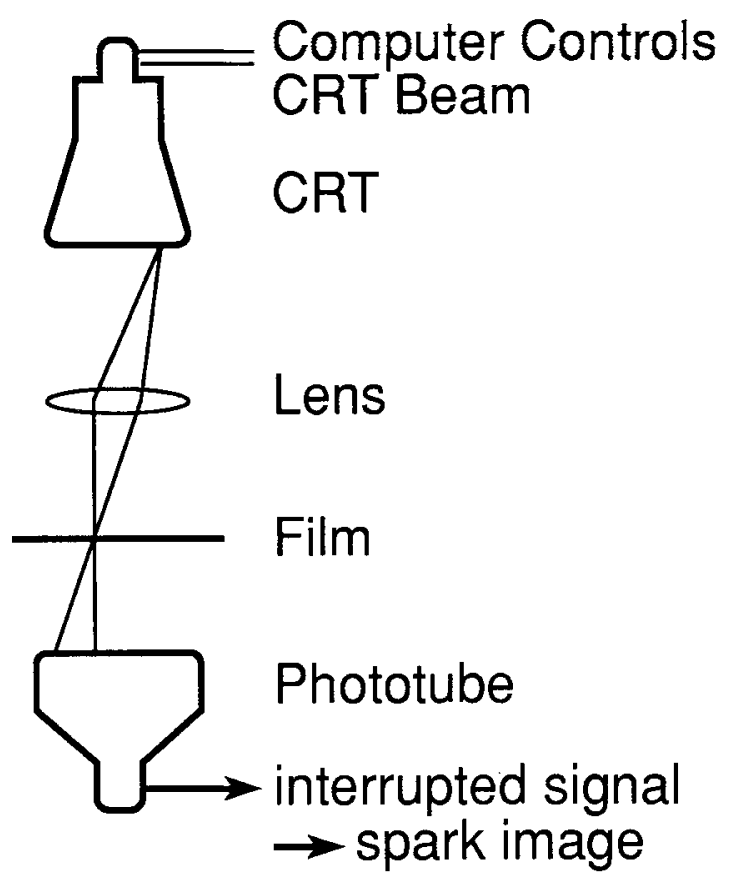

Fig. 19a. The autoscan system.

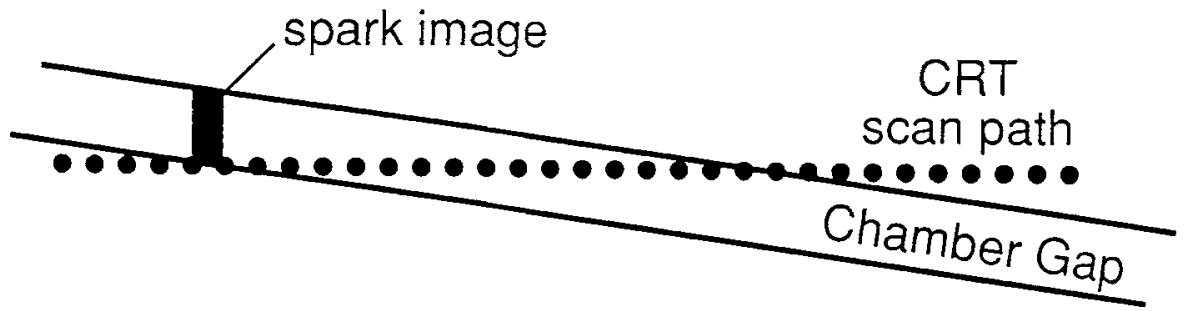

Fig. 19b. Illustration that random film rotations could cause the CRT scan path to leave a chamber gap. This was a serious problem because of the large demagnification from about $8 \mathrm{ft}$. in real space down to $25 \mathrm{~mm}$. on film. 
computer control program sufficed. This did not work for the $N^{*}$ experiment essentially because we could not keep a straight line CRT scan in the center of a gap as illustrated in Fig. 19b. In this case, because of the large demagnification needed to fit the chamber images into $35 \mathrm{~mm}$, a chamber gap on the film was only about 7 CRT spot sizes wide. Random rotations of the film as it was being clamped in place for each frame could cause the scan line to leave the chamber gap as indicated. To compensate for this, it was necessary, for each frame, to locate the fiducials, and then relative to these to create a scan path for the CRT that would keep the spot in the center of a chamber gap. In order to carry out these more complicated operations we needed a more powerful computer than was used in the earlier experiment. Kent took the lead in selecting an the appropriate computer, ordering it and making it work. Making it work was not a simple problem; it took several years to solve completely.

\section{WHAT WE LEARNED FROM THE $N^{*}$ and $\rho$ EXPERIMENTS}

Finally, I will address the question of what we learned from these experiments. For the purposes of this discussion, I think that is most interesting to see if our results had an impact on later work, and if so, what it was. I therefore asked our librarian, Jack Weigel, to do a literature search for references to our papers. The data base that he used went back only to 1974, several years after our publications, so the survey gives only a lower limit to the number of citations. I will present the results of the literature search shortly, but first I must mention how the topics in the $N^{*}$ experiment were divided up for theses and for publication.

Elastic scattering was one obvious topic and this was chosen by Ed Meanley. We did indeed find backward production of a particular $N^{*}$ resonance, the $\Delta(1236), I=\frac{3}{2}, J=\frac{3}{2}$ resonance, as I will show in a moment. This topic was taken by Jim Rice. In addition, the continuum distribution of high mass states proved to be interesting and this was Bob Anthony's thesis. I must add also, that we were joined by another student, Lou Byerly, when the analysis of the $N^{*}$ experiment was well along, and he took on the $\rho$ data which had not been touched until that point.

With this division of topics, the results of the literature search were as shown in the table on the next page. 


\begin{tabular}{lllc} 
Topic & Student & Date & Citations \\
\cline { 2 - 3 }$\Delta(1236)$ & Jim Rice & 1970 & 4 \\
Continuum & Bob Anthony & 1971 & 2 \\
Elastic & Ed Meanley & 1970 & 1 \\
$\rho$ & Lou Byerly & 1970 & 0
\end{tabular}

It is not surprising that the $\rho$ experiment was not particularly popular. In the interval between conception and completion of our experiment, the era of large bubble chamber collaborations had begun. Of the two $\rho$ final states studied in our experiment, $\rho^{0}$ and $\rho^{-}$, only for the latter did we have statistical power comparable to that of a number of bubble chamber experiments and only this channel was analyzed. ${ }^{7}$

I will comment further only on backward $\Delta$ production. My remarks are intended to illustrate the kinds of analyses of strong reactions that were in favor in the late 60' and early 70's. In discussing Jim Rice's results I will incorporate some data from later experiments that cited ours.

As I mentioned above, we did see backward production of the 1236, $\left(\frac{3}{2}, \frac{3}{2}\right) \Delta$ resonance in the reaction $\pi^{-} p \rightarrow \Delta^{-} \pi^{+}$. Before discussing the analysis of this reaction, I must add a bit of history to explain the variables that we used to describe our data.

In the few years between our proposal and the analysis of our results, the concept of baryon exchange as a mechanism for producing a backward peak had become well accepted. This was because of an apparently perfect correlation between the presence of substantial backward production and the presence of a diagram involving the exchange of a particle having the quantum numbers ( charge, isospin and strangeness) of a known baryon. ${ }^{8}$ Backward production of $\Delta^{-} \pi^{+}$is then expected to go by exchange of either a neutron or a $\Delta^{0}$, while backward production of $\Delta^{+}$would go by exchange of a $\Delta^{++}$as illustrated in Fig. 20 below.

Fig. 20.

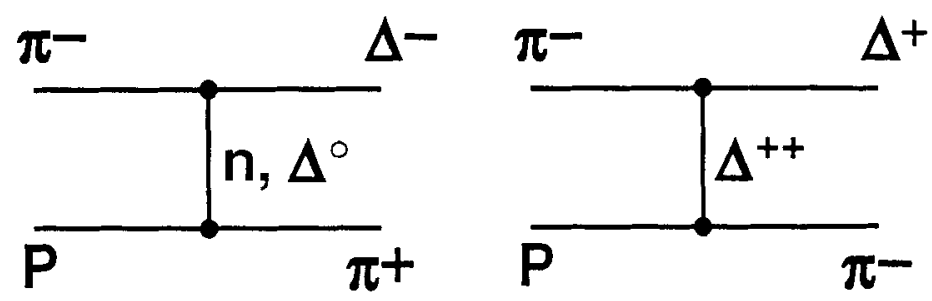

The variable that appears naturally in the discussion of these 
diagrams is the square of the four momentum transfer from incident to final baryon,

$$
u=\left(p_{\Delta}-p_{p}\right)^{2} .
$$

We actually used a variable $u^{\prime}$ defined by

$$
u^{\prime} \equiv u-u_{180^{\circ}}=-2 p_{0}^{*} p^{*}\left(1+\cos \theta^{*}\right),
$$

where $p_{0}^{*}$ and $p^{*}$ are initial and final center of mass momenta, and $\theta^{*}$ is the center of mass production angle. The virtue of $\mathrm{u}$ as angle variable rather than $\theta^{*}$ is that with $\mathrm{u}$, one expects the shape of the differential cross section to vary slowly with beam momentum and this turns out to be true.

Now, returning to $\Delta$ production, our data, as well as data from another experiment, is shown on the next three pages. Fig. 21 is an example of one of our mass distributions ${ }^{9}$ for which the $\Delta^{-}(1236)$ is clearly visible. Similar plots for the other $\Delta$ channel, $\pi^{-} p \rightarrow \Delta_{1236}^{+} \pi^{-}$, showed hints of a signal, but not enough for any interesting analysis.

Notice that in these mass plots, there is no clear $\Delta$ production for the smallest values of $\left|u^{\prime}\right|$ near the backward direction. We were therefore seeing a backward valley rather than a backward peak. This can be seen more clearly in the differential cross sections of Fig. 22. which give $d \sigma / d u^{\prime}$ vs $u^{\prime}$. The straight lines are linear fits to the data and are of no particular interest. This data by itself would not be very useful. There is a valley, but what is it a valley in? Fortunately, bubble chamber data ${ }^{10,11}$ that covered essentially all angles, at the same beam momenta as our two lowest beam momenta, was available. Fig. 23 shows the differential cross section for $\pi^{-} p \rightarrow \Delta^{-} \pi^{+}$at $2.7 \mathrm{GeV}$ from one of these bubble chamber experiments ${ }^{11}$. There is indeed a backward peak and what we were seeing was a very sharp valley on the top of the peak. I will show this data plotted together with ours in a moment.

The references to our papers that I mentioned earlier were all from reports on later experiments ${ }^{12,13,14}$ studying baryon exchange, most of them done at higher momenta than ours. These experiments all have the same general character shown in Fig. 24. In these experiments, events were triggered on very forward decay products of the $\Delta$, and detectors surrounding the target permitted direction and momentum measurements on all the charged particles. 
154 Strong Interaction Experiments at the ZGS

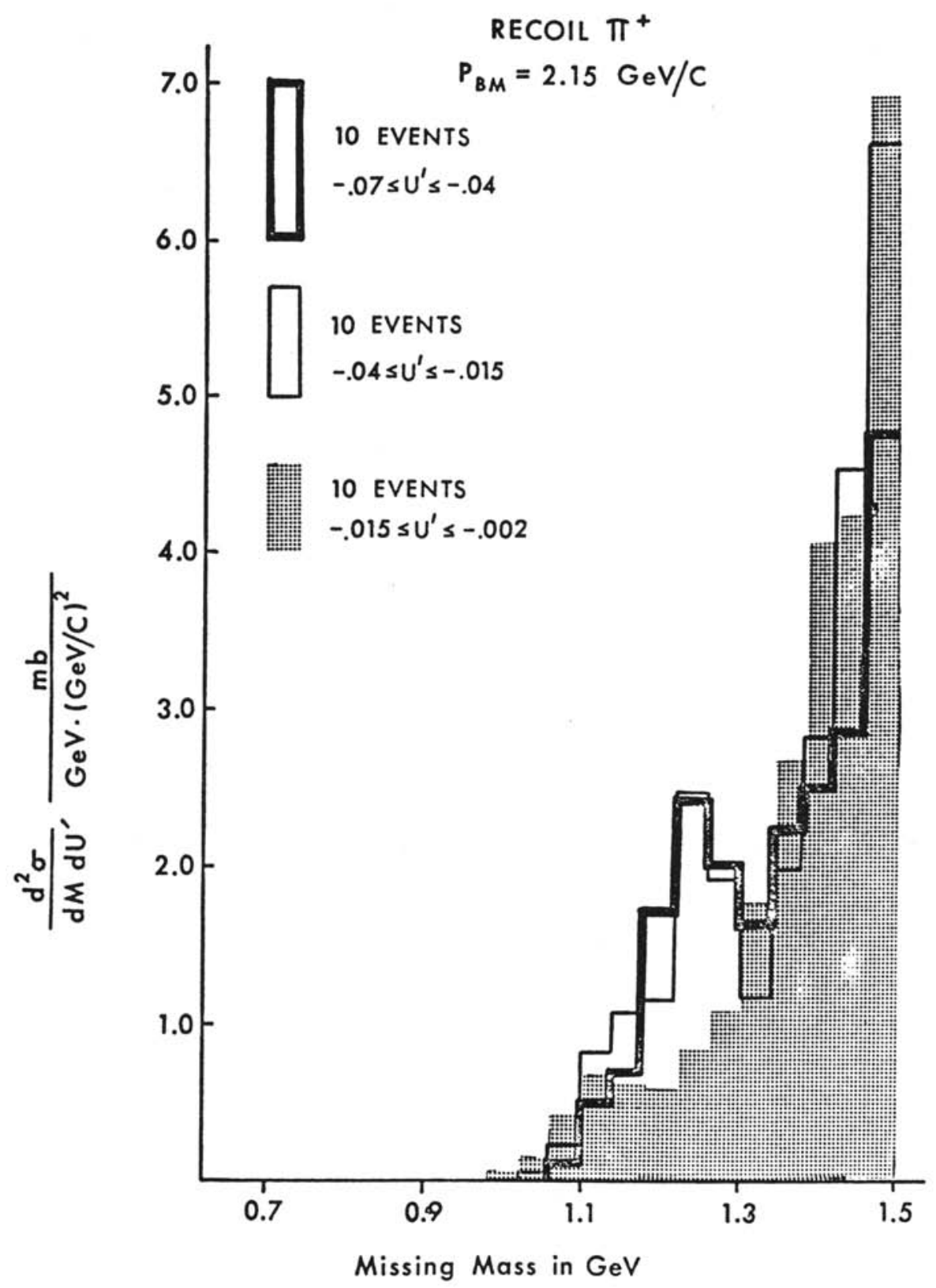

Fig. 21. Missing mass spectra at $2.15 \mathrm{GeV}$ for recoil $\pi^{+}$. Shown are three spectra for different intervals in $u^{\prime}=u-u_{\max }$. Note the absence of a $\Delta$ signal for the shaded histogram corresponding to the most backward direction. Our data, are taken from Ref. 9 . 
C. Tristram Coffin 155

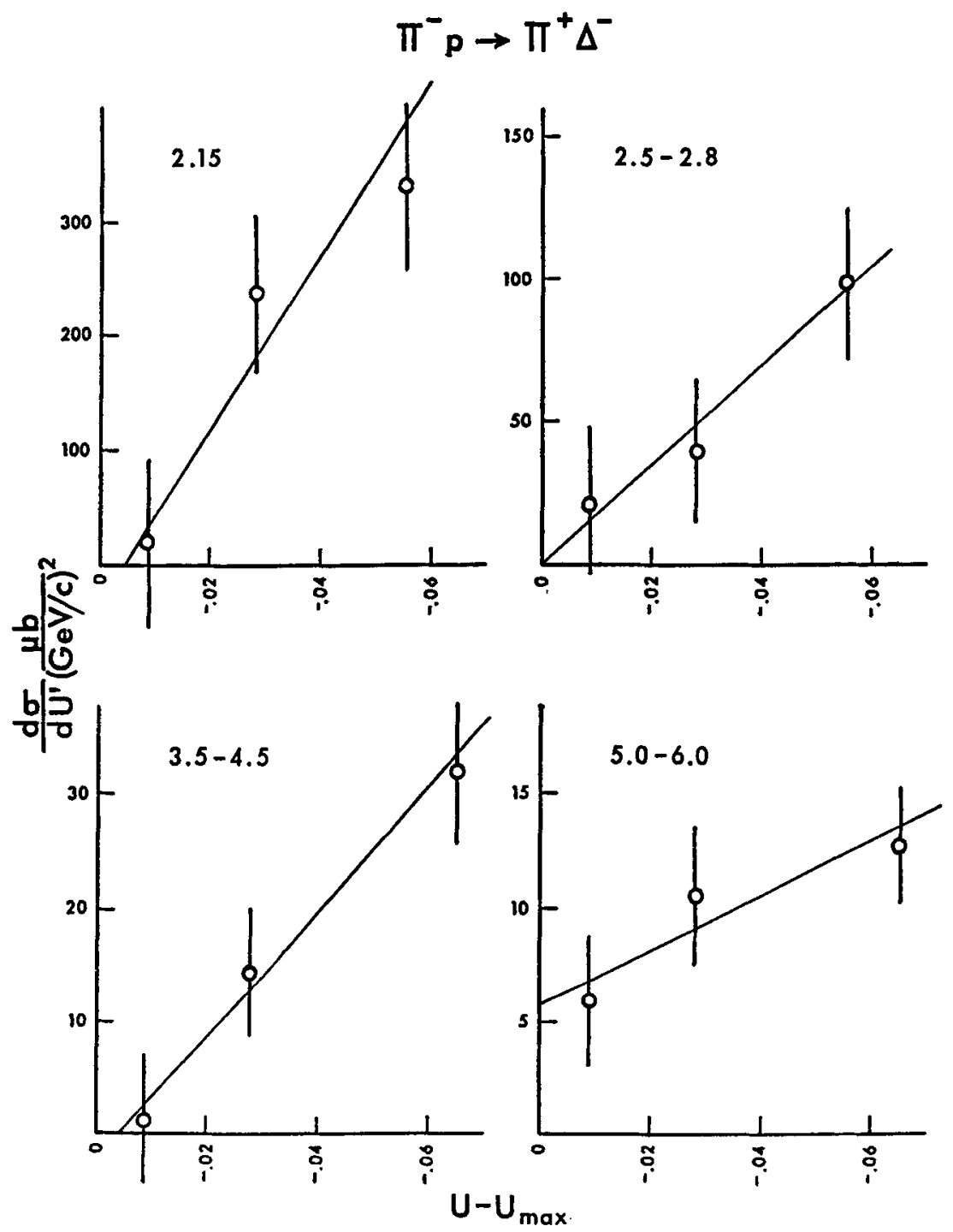

Fig. 22. Differential cross sections for $\pi^{-} p \rightarrow \Delta^{-} \pi^{+}$near the backward direction ( from Ref. 9 ). 
156 Strong Interaction Experiments at the ZGS

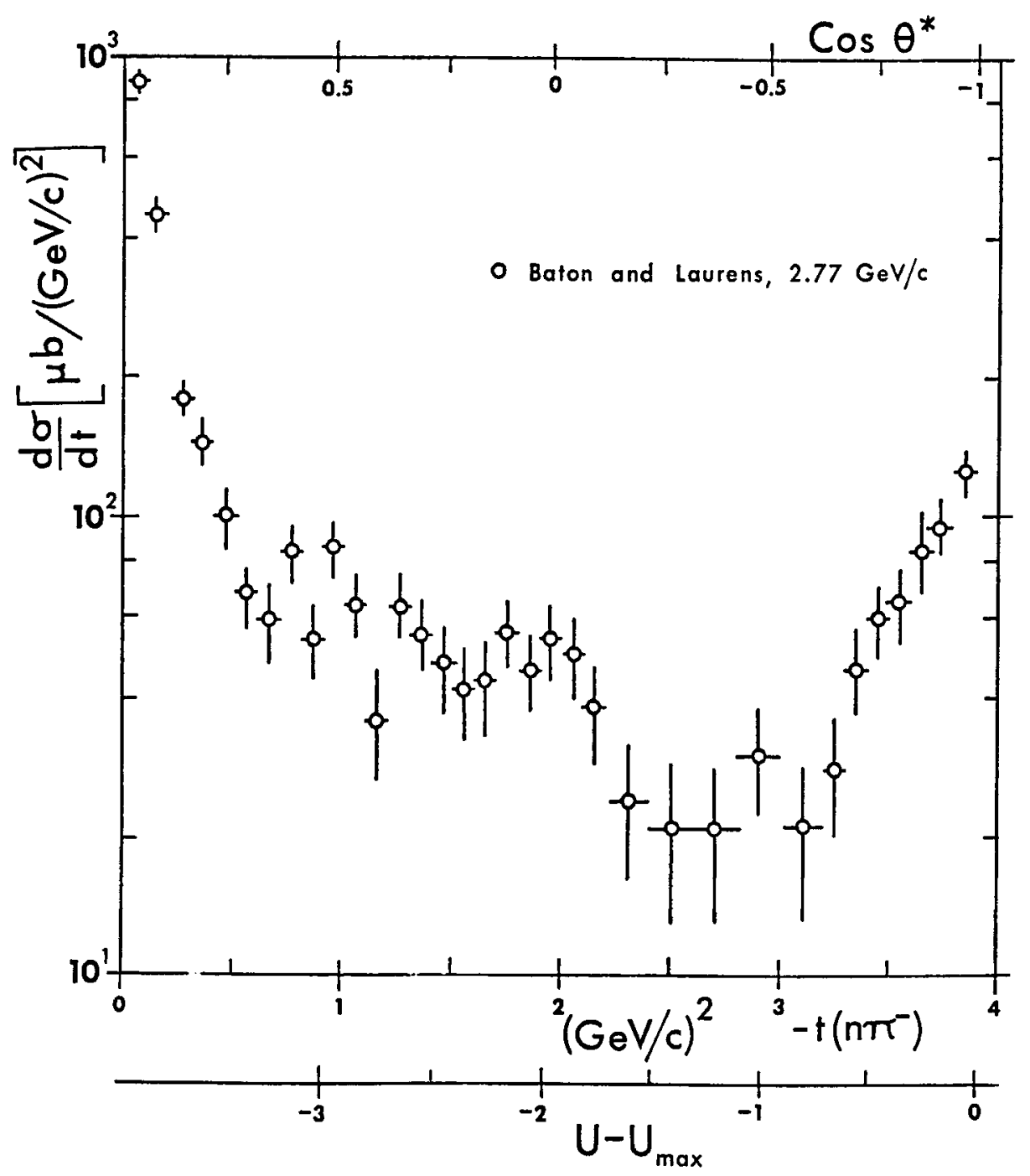

Fig. 23. Differential cross section for $\pi^{-} p \rightarrow \Delta^{-} \pi^{+}$at $2.77 \mathrm{Gev}$ from the bubble chamber experiment of Ref. 11 . 


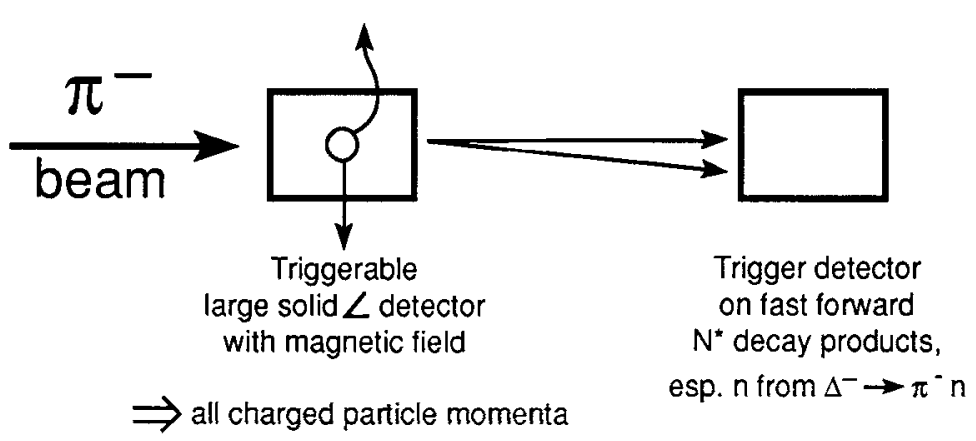

Fig. 24. General features of the backward $N^{*}$ experiments of Refs. 12,13 and 14. An event trigger relies heavily on detecting very forward $N^{*}$ decay products, while kinematic information on other particles is provided by visual detectors surrounding the target.

Fig. 25 on the next page includes, I believe, all existing data on backward production of $\Delta^{-} \pi^{+}$. Our data covers only the backward valley for beam momenta from 2 to $6 \mathrm{GeV}$. The other experiments fix the behavior at larger angles. The 1978 ZGS experiment at 8 $\mathrm{GeV}$ is the only one of the large angle experiments with enough statistical power to see both the valley and peak in a single experiment.

I have taken some artistic liberty with this data by drawing the same freehand curve through all of the points. It seems very likely that this process exhibits essentially a fixed structure in $u^{\prime}$ at all beam momenta. The sharp backward valley is a rather unique effect in baryon exchange reactions.

I have noted on Fig. 25 the special value $u^{\prime}=-0.055 \mathrm{GeV}^{2}$. This is the value of $u^{\prime}$ that we chose in studying the energy dependence, and other later experimenters have used the same value. Fig. 26 shows such an energy dependence in $d \sigma / d u^{\prime}$ at fixed $u^{\prime}$ for all of the data in Fig. 25. In Fig. 26, the energy variable is $s$, the square of the production frame center of mass energy. The power law fit gives an $s^{-4}$ dependence. I have shown this graph primarily to illustrate the longevity of the study of baryon exchange. This graph comes from a 1982 paper by a Russian group at ITEP $^{14}$ who used a solid deuterium target surrounded by op- 
158 Strong Interaction Experiments at the ZGS

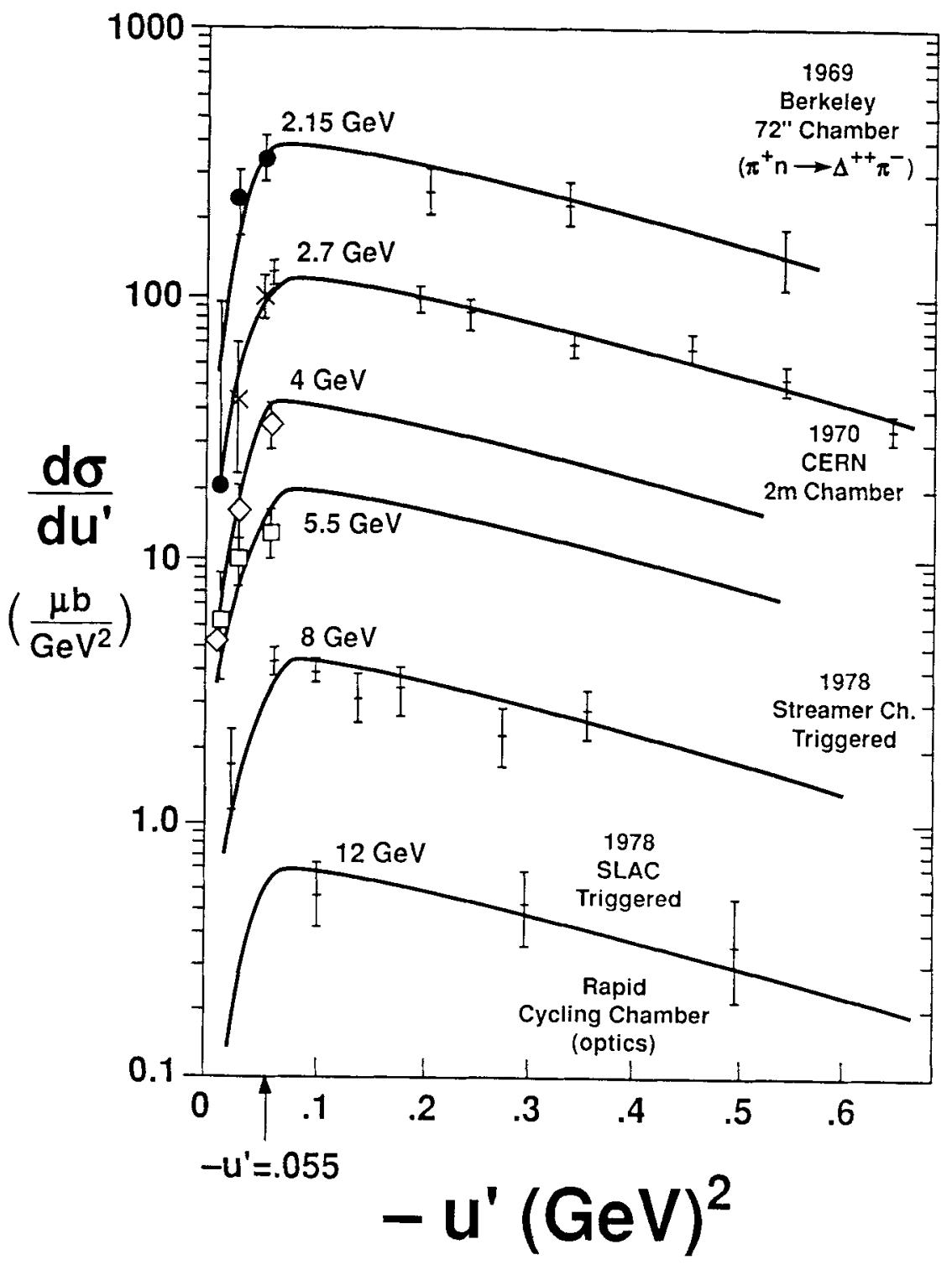

Fig. 25. Summary of differential cross sections for backward production in $\pi^{-} p \rightarrow \Delta^{-} \pi^{+}$. The large angle data comes from: 2.15 GeV-Ref.10, 2.77 Gev-Ref.11, 8 GeV-Ref. 12, 12 GeV-Ref. 13. 
C. Tristram Coffin 159

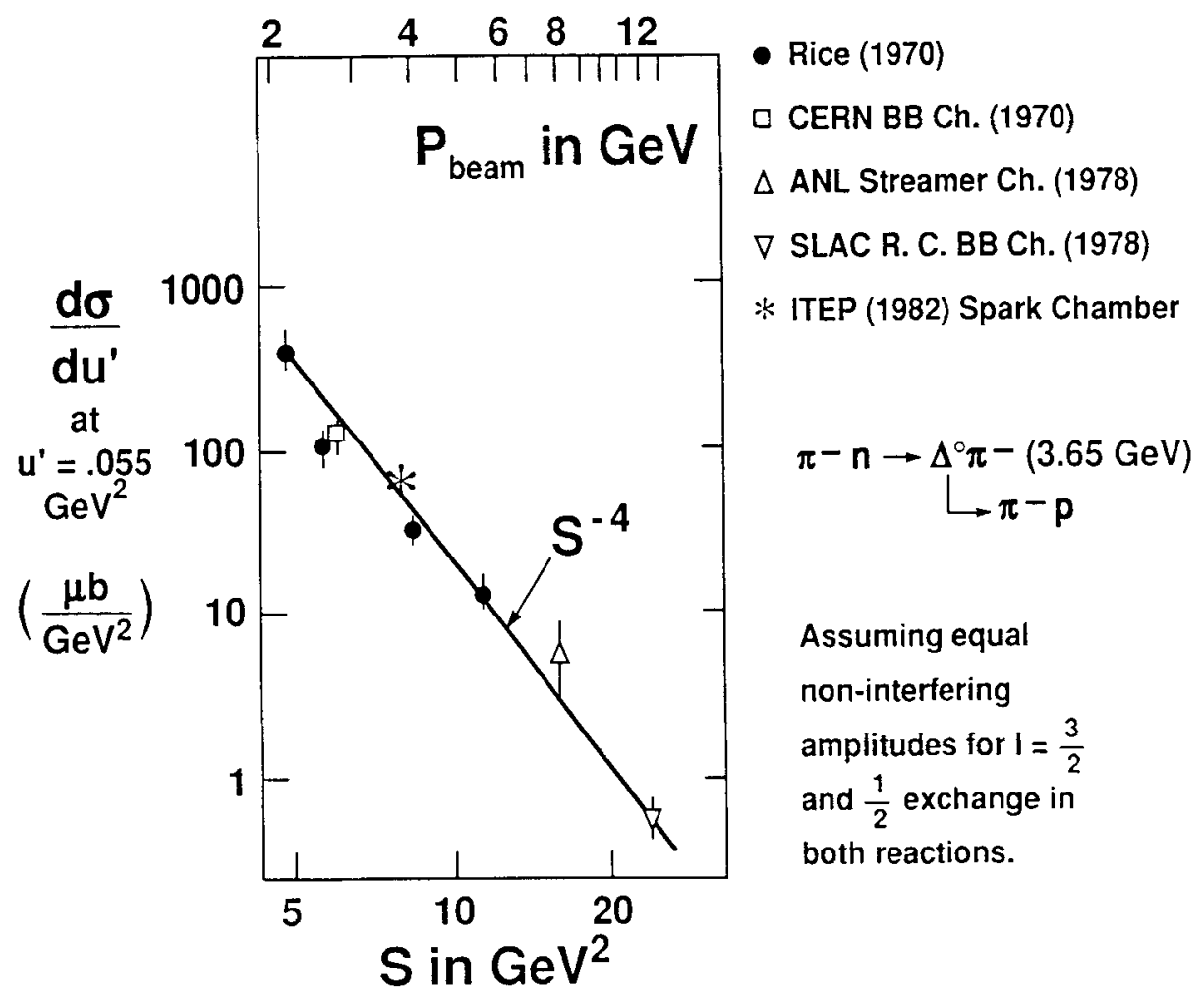

Fig. 26. Energy dependence of the differential cross section, $d \sigma / d u^{\prime}$ at $u^{\prime}=-0.55 \mathrm{GeV}^{2}$ for $\pi^{-} p \rightarrow \Delta^{-} \pi^{+}$. The starred point at $s=7 G e v^{2}$ is inferred from data on $\pi^{-} n \rightarrow \Delta^{0} \pi^{-}$( from isospin arguments ) by the authors of Ref. 14 . 
tical spark chambers to study the reaction $\pi^{-} n \rightarrow \Delta^{0} \pi^{-}$. Their cross section cannot be compared directly with the others on the graph because their process involves a different combination of the allowed baryon exchange amplitudes, $I=\frac{3}{2}$ and $I=\frac{1}{2}$, than those needed for $\pi^{-} p \rightarrow \Delta^{-} \pi^{+}$. With special assumptions about these two amplitudes they infer a cross section for our process consistent with the trend of the other data.

I summarize this discussion of $\Delta$ production by remarking that it is a very nice effect that has yet to receive any serious theoretical study as nearly as I can tell. It seems unlikely that it ever will be given attention by theorists unless there is some new development in nonperturbative models of strong reactions.

Finally I note that whether or not the data from this experiment is ever interpreted theoretically, it generated considerable interest. It was fortunate for all of us that Kent took the forceful stand that he did on that train ride from Ann Arbor to Chicago because the $\rho$ experiment turned out to be pretty pedestrian.

\section{REFERENCES}

1. C. Alff, D. Berley, D. Colley, N. Gelfand, U. Nauenberg, D. Miller, J. Schultz, J. Steinberger, and T.H. Tan, Phys. Rev. Letters $\underline{9}, 322$ (1962).

2. J. D. Jackson, Rev. Mod. Phys. $\underline{37}, 484$ (1965).

3. Aachen-Berlin-Birmingham-Bonn-Hamburg-London(I.C.) -Munchen Collaboration, Phys. Rev. 138, B 897 (1965).

4. S. W. Kormanyos, A. D. Krisch, J. R. O'Fallon and K. Ruddick, Phys. Rev. 164, 1661 (1967).

5. C. T. Coffin, N. Dikmen, L. Ettlinger, D. Meyer, A. Saulys, K. Terwilliger, and D. Williams, Phys. Rev. 159, 1169 (1967)

6. V. Barger and D. Cline, Phys. Rev. Letters 16, 913 (1966).

7. L. Byerly, R. Anthony, C. T. Coffin, E. Meanley, D. Meyer, J. Rice, N. R. Stanton, and K. M. Terwilliger, Phys. Rev. D I, 637 (1973).

8. V. D. Barger and D. B. Cline, Phenomenological Theories of High Energy Scattering ( Benjamin, New York, 1969 ).

9. J. E. Rice, R. W. Anthony, C. T. Coffin, E. S. Meanley, and K. M. Terwilliger, Phys. Rev. Letters 27, 687 (1971).

10. P. M. Dauber, P. Hoch, R. J. Manning, D.M. Siegel, M. A. Abolins and G. A. Smith, Phys. Letters 29B, 609 (1969). 
11. J. P. Baton and G. Laurens, Nucl. Phys. B21, 551 (1970)

12. A. Abashian, B. Eisenstein, J. D. Hansen, W. Mollet, B. Nelson, T. O'Halloran, J. R. Orr, D. Rhines, P. Schultz, P. Sokolsky, R. G. Wagner, J. Watson, N. M. Gelfand, and M. Buttram, Phys. Rev. D $\underline{13}, 5$ (1976).

13. M. W. Arenton, W. J. Bacino, J. M. Hauptman, F. D. Rudnick, P. F. Shepard, W. E. Slater, D. H. Stork, and H. K. Ticho, Nucl. Phys. B141, 77 (1978)

14. A. V. Aref'ev, G.A. Leksin, D. A. Suchkov and

F. M. Khasanov, Sov. J. Nucl. Phys. 35(6), 847 (1982) 